\title{
1 Relation between Type II Bursts and CMEs \\ 2 Inferred from STEREO Observations
}

\author{
3 N. GOPALSWAMY, W. THOMPSON, J. DAVILA, M. L. KAISER \\ $4 \quad$ NASA Goddard Space Flight Center, Greenbelt, MD \\ 5 S. YASHIRO \\ 6 Interferometrics, Herndon, VA \\ 7 P. MÄKELÄ, G. MICHALEK \\ 8 The Catholic University of America, Washington, DC \\ 9 J.- L. BOUGERET \\ 10 Paris Observatory, Meudon, France \\ 11 R. A. HOWARD \\ 12 Naval Research Laboratory, Washington, DC

14 Abstract

15 The inner coronagraph (COR1) of the Solar Terrestrial Relations Observatory (STEREO) mission

16 has made it possible to observe CMEs in the spatial domain overlapping with that of the metric

17 type II radio bursts. The type II bursts were associated with generally weak flares (mostly B and C

18 class soft X-ray flares), but the CMEs were quite energetic. Using CME data for a set of type II

19 bursts during the declining phase of solar cycle 23, we determine the CME height when the type II

20 bursts start, thus giving an estimate of the heliocentric distance at which CME-driven shocks form.

21 This distance has been determined to be $\sim 1.5 \mathrm{Rs}$ (solar radii), which coincides with the distance at

22 which the Alfven speed profile has a minimum value. We also use type II radio observations from

23 STEREO/WAVES and Wind/WAVES observations to show that CMEs with moderate speed

24 drive either weak shocks or no shock at all when they attain a height where the Alfven speed peaks

25 ( $-3-4$ solar radii). Thus the shocks seem to be most efficient in accelerating electrons in the

26 heliocentric distance range of 1.5 to 4 Rs. By combining the radial variation of the CME speed in

27 the inner corona (CME speed increase) and interplanetary medium (speed decrease) we were able

28 to correctly account for the deviations from the universal drift rate spectrum of type II bursts, thus confirming the close physical connection between type II bursts and CMEs. The average height $(\sim 1.5 \mathrm{Rs})$ of STEREO CMEs at the time of type II bursts is smaller than that (2.2 Rs) obtained for SOHO (Solar and Heliospheric Observatory) CMEs. We suggest that this may indicate, at least partly, the density reduction in the corona between the maximum and declining phases, so a given plasma level occurs closer to the Sun in the latter phase. In two cases, there was diffuse shock-like feature ahead of the main body of the CME, indicating a standoff distance of 1-2 Rs by the time the CME left the LASCO FOV.

KEY WORDS:

38 Coronal mass ejections; type II radio bursts; shocks; flares; dynamic spectrum

\section{Introduction}

41 One of the major disadvantages in studying the physical relation between coronal mass ejections (CMEs) and metric type II radio bursts has been the lack of spatial overlap between the observations. Since a type II burst is the earliest signature of 
1 a shock, identifying the CME leading edge at the time of the metric type II burst

2 provides an estimate of the height at which the shock forms. Knowing the

3 heliocentric distance at which the shock forms is important in understanding many

4 aspects of CMEs such as the charge state composition of solar energetic particles

5 observed in the IP medium. Using the height - time plots of white-light CMEs

6 observed by the Large Angle and Spectrometric Coronagraph (LASCO,

7 Brueckner et al., 1995) on board the Solar and Heliospheric Observatory (SOHO)

8 mission, Gopalswamy et al. (2005) found an average CME height of $\sim 2.2 \mathrm{Rs}$ at

9 the onset of metric type II bursts. This result was based on type II bursts

10 originating from close to the limb (central meridian distance, CMD between $60^{\circ}$

11 and $90^{\circ}$ ), so the projection effects are minimal and hence the height measurements

12 are accurate. Unfortunately, the SOHO/LASCO occulting disk obscured the

13 corona to a heliocentric distance of $\sim 2.5$ Rs. This means type II bursts typically

14 start when the CMEs are still below the occulting disk. Therefore, the CME

15 height-time plots from SOHO/LASCO have to be extrapolated to the times of type

16 II bursts to estimate the CME heights. The expanded field of view (FOV) of the

17 inner coronagraph (COR1) of the Sun Earth Connection Coronal and Heliospheric

18 Investigation (SECCHI) instrument on board the Solar TErrestrial RElations

19 Observatory (STEREO) mission has changed this situation by providing CME

20 height measurements down to 1.4 Rs. This is an important new capability for type

21 II burst studies because the COR1 FOV overlaps with the spatial domain of the

22 corona where the type II bursts start (Gopalswamy and Kaiser, 2002).

23 Furthermore, the outer coronagraph (COR2) on SECCHI overlaps with the

24 LASCO FOV for consistency check. The present study makes use of the COR1

25 capability to determine CME heights at the onset of type II bursts without

26 extrapolation.

27 A related problem is the evolution of the CME in the inner corona. Since the CME

28 starts from rest as it erupts, it undergoes rapid acceleration in the beginning,

29 reaches a maximum acceleration in the inner corona before being controlled by

30 the aerodynamic drag (Wood et al., 1999; Gopalswamy and Thompson, 2000;

31 Zhang et al., 2001; Vrsnak, 2001). The CME acceleration occurs at a heliocentric

32 distance where the characteristic speed in the corona attains a minimum in the

33 periphery of active regions (Gopalswamy et al., 2001a; Mann et al., 2003) before

34 reaching a peak in the outer corona (Mann et al., 1999; Gopalswamy et al., 
1 2001a). Since the CME speed needs to exceed the characteristic speed before it

2 can drive a shock, the combination of CME acceleration and the rapid change in

3 the fast mode speed produces interesting possibilities for shock formation and

4 decay (Gopalswamy et al., 2001a). STEREO/COR1 provides CME measurements

5 in this important spatial domain and hence can capture the early evolution of

6 CMEs. In addition, SECCHI's Extreme Ultraviolet Imager (EUVI) obtains high

7 cadence images providing information on the $\mathrm{CME}$ and the surrounding EUV

8 waves very close to the solar surface.

9 Information on the early evolution of CMEs has important implications for

10 understanding the spectral evolution of type II bursts throughout the inner

11 heliosphere. It is well known that the frequency drift rate of type II bursts in the

12 radio dynamic spectra are related to the speed of the shock that produces the

13 bursts and the density gradient in the ambient medium. It was noted by several

14 authors that the frequency drift rate is a power law in the frequency of emission

15 (Mann et al., 1996; Vrsnak et al., 2001; Vrsnak et al., 2002; Aguilar-Rodriguez et

16 al., 2005). The power law was found to be valid when observations from different

17 spectral domains [metric (m), decameter-hectometric (DH), and kilometric $(\mathrm{km})$

18 wavelengths] are combined for the same events or for different events. Similarly,

19 the power law was maintained when observations from different instruments and

20 different epochs are combined. This universal nature of the power law suggests

21 that the same shock should be involved in producing radio bursts at various

22 spectral domains. In the spatial domain, this means the propagation of CME-

23 driven shocks starting near the Sun and propagating far into the IP medium

24 (Gopalswamy, 2006b). One of the assumptions we make in this paper is that the

25 heliocentric distance of the type II burst (and of the shock) can be approximated

26 by that of the driving CME. We also assume that the shock speed is similar to the

27 CME speeds. The shock is expected to surround the CME as a bow wave at the

28 standoff distance. Near the Sun, the standoff distance may not be too large. On the

29 other hand, the electrons responsible for the type II burst may be accelerated at the

30 shock flanks (Holman and Pesses, 1983), which means the heliocentric distance of

31 the type II burst location may be smaller than the CME leading edge. Thus, the

32 CME height is a reasonable approximation to the heliocentric distance of the

33 shock, especially because of the lack of spatial information for the type II burst.

34 The analyses in this paper adheres to one of the paradigms of type II radio bursts 
1 that CME-driven shocks can explain type II bursts in all wavelength domains. We

2 do appreciate that there is alternative paradigm that flare blast waves produce

3 some metric type II bursts (see Vrsnak and Cliver, 2008 for a review on this

4 topic). We shall show that the data and results presented in this paper are more

5 consistent with the CME-driven shock paradigm.

7 We study all the metric type II radio bursts and their interplanetary counterparts

8 observed since the launch of the STEREO mission, in conjunction with the CMEs

9 and flares associated with the bursts. In section 2, we describe the radio and

10 optical data used in this paper and arrive at some overall conclusions about the

11 type II bursts and the associated CMEs. In section 3, we describe four type II

12 bursts and the associated CMEs in detail and connect the CME speed evolution to

13 the duration of the type II burst by addressing the parallel evolution of the Alfven

14 speed profile in the corona. In one event, the type II burst was observed in the

15 interplanetary (IP) medium, which we connect to the CME evolution in the outer

16 corona. In section 4 we show that the deviations from the universal drift rate

17 spectrum of type II bursts can be explained by the opposite tendencies in the

18 radial variation of CME speed in the metric and IP domains of type II radio bursts.

19 Section 5 discusses the results and provides a summary of the results.

21 Table 1. Spatial resolution and field of view of instruments from SOHO and

22 STEREO used in this paper.

\begin{tabular}{lrrrr}
\hline & Pixel Size & CCD Size & FOV & Cadence \\
\hline EIT 195 & $2.6^{\prime \prime}$ & $1024 \times 1024$ & $0-1.4 \mathrm{Rs}$ & $12 \mathrm{~min}$ \\
LASCO C2 & $11.2^{\prime \prime}$ & $1024 \times 1024$ & $2.1-6.0 \mathrm{Rs}$ & $\sim 20 \mathrm{~min}$ \\
LASCO C3 & $56.0^{\prime \prime}$ & $1024 \times 1024$ & $3.7-30 \mathrm{Rs}$ & $\sim 30 \mathrm{~min}$ \\
SECCHI EUVI & $1.6^{\prime \prime}$ & $2048 \times 2048$ & $0-1.7 \mathrm{Rs}$ & $2.5 \mathrm{~min}$ \\
SECCHI COR1 & $3.75^{\prime \prime}$ & $2048 \times 2048$ & $1.4-4 \mathrm{Rs}$ & 5 or $10 \mathrm{~min}$ \\
& $\left(7.5^{\prime \prime}\right)$ & $(1024 \times 1024)$ & & \\
SECCHI COR2 & $14.7^{\prime \prime}$ & $2048 \times 2048$ & $2-15 \mathrm{Rs}$ & $15 \mathrm{~min}$ \\
\hline
\end{tabular}

\section{2. Data Description and Overview}

25 We collected information on type II radio bursts that occurred during January

262007 to November 2008 from two main sources: (i) the list of metric type II radio 
1 bursts archived at the National Geophysical Data Center (NGDC) and available

2 online

3 (ftp://ftp.ngdc.noaa.gov/STP/SOLAR_DATA/SOLAR_RADIO/SPECTRAL) and

4 at the on-line Solar Geophysical Data; (ii) list of type II bursts in the DH and km

5 wavelengths detected by the Radio and Plasma Wave Experiments on board Wind

6 and STEREO (Bougeret et al., 1995, 2008) available at

7 http://lep694.gsfc.nasa.gov/waves/waves.html. We also compared the two lists to

8 see if any of the metric type II bursts had DH counterparts and vice versa. For

9 each type II burst, we identified the associated CME from STEREO and SOHO

10 instruments that image the corona in EUV and/or white light. The COR1 FOV

11 extends from 1.4 to 4 Rs, but when combined with the SECCHI/EUVI and the

12 extreme-ultraviolet Imaging Telescope (EIT) on board SOHO, we can observe the

13 CMEs all the way to the surface. The combined data set thus provides an

14 opportunity to track CMEs and the associated disturbances up to a heliocentric

15 distance of 32 Rs from close to the solar surface. The SECCHI heliospheric

16 imagers extend the FOV all the way to Earth orbit, but we do not use the data in

17 this study because most of the type II bursts were confined to frequencies that

18 correspond to the near-Sun part of the interplanetary medium. Table 1 shows the

19 instrument capabilities and fields of view (see Howard et al., 2008 for more

20 details).

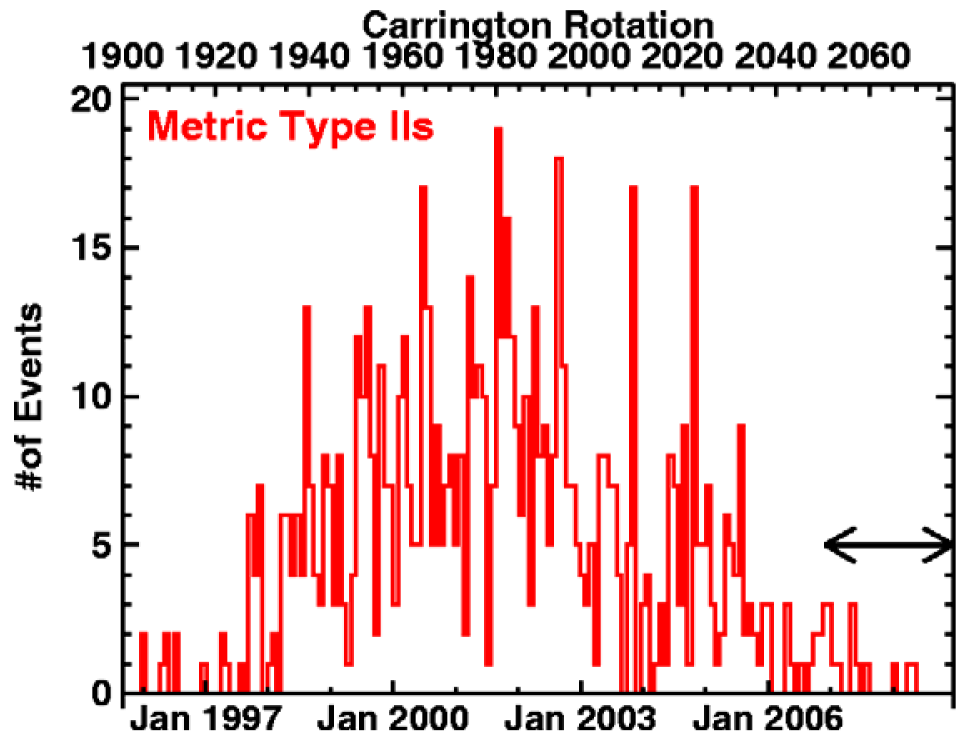

22 Figure 1. Occurrence rate of metric type II bursts binned over Carrington

23 rotation periods from 1996 to November 2008. The study period is indicated by 
1 the double arrow. The Carrington rotation numbers are shown in the top.

2 During solar maximum, the highest rate was 19 type II bursts per rotation.

4 The study period is at the declining phase of the solar cycle 23 with very low level

5 of solar activity (see Fig. 1). This has the advantage of making unambiguous

6 identification of the solar eruption associated with each type II radio burst. The

7 type II bursts were well isolated and all but one originated from numbered active

8 regions. The single exception is the type II burst on 2008 April 26 originating

9 from an unnumbered active region. By playing Javascript movies of EUV and

10 white-light images, we were able to identify the EUV disturbances in the active

11 region, followed by a white-light CME in the inner and outer coronal regions. The

12 larger-scale EUV disturbance is typically seen as a global wave-like phenomenon

13 observed around the active region, distinctly larger than the bright flare loops or

14 post-eruption arcades. The outermost part of the disturbance is often the EUV

15 wave that surrounds the CME. The CME itself is occasionally observed in EUVI

16 as a loop-like structure that can be identified with the erupting flux rope

17 subsequently observed in white light. Examples of such cases will be presented

18 'later. Each eruption is also identified with a soft X-ray flare, whose details can be

19 found in the on-line SGD or NGDC flare archive. The heliographic coordinates

20 of the soft X-ray flare in the active region serves as the solar source location of the

21 eruption. We refer to the heliographic coordinates from Earth point of view

22 (which is the same as for a spacecraft along the Sun-Earth line, such as SOHO).

23 Table 2 lists the metric type II bursts investigated in this paper. The date

$24 \quad(\mathrm{yy} / \mathrm{mm} / \mathrm{dd}$ format) and time range ( $\mathrm{hh}: \mathrm{mm}-\mathrm{hh}: \mathrm{mm}$ format) of the metric type II

25 bursts are listed in columns 1 and 2 , respectively. The starting $\left(f_{s}\right)$ and ending $\left(f_{e}\right)$

26 frequencies of the type II bursts are given in column 3. Note that the ending

27 frequency usually means the burst reaching the lower frequency end of the

28 dynamic spectrum. A question mark means we are not sure whether the emission

29 is at the fundamental or harmonic of the plasma frequency. The drift rate $(\mathrm{MHz} / \mathrm{s})$

30 in the metric range obtained from the dynamic spectra available at NGDC is given

31 in column 4. When the type II burst continues to decameter-hectometric (DH)

32 wavelengths, the ending time is given in column $5 ; \mathrm{N}$ indicates no $\mathrm{DH}$

33 component. Only one (2007 January 25) type II burst had extended to the $\mathrm{km}$

34 domain. The speed of the CMEs as measured within the COR1 FOV ( $V_{C}$, column 
1 6) and LASCO FOV ( $\mathrm{V}_{\mathrm{L}}$, column 7) is based on a linear fit to the height-time

2 measurements in the respective FOVs. The CME width as measured in the

3 LASCO FOV is given in column 8. The CME information in columns 7 and 8 are

4 Table 2. List of metric type II bursts and the properties of CMEs.

\begin{tabular}{|c|c|c|c|c|c|c|c|c|c|}
\hline Date & $\begin{array}{l}\text { Time } \\
\text { range }\end{array}$ & $\begin{array}{l}f_{e}-f_{s} \\
\text { (MHz) }\end{array}$ & $\begin{array}{l}\mathrm{df} / \mathrm{dt} \\
\mathrm{MHz} / \mathrm{s}\end{array}$ & $\begin{array}{l}\text { DH } \\
\text { II }\end{array}$ & $\begin{array}{l}V_{c} \\
\mathbf{k m} / \mathbf{s}\end{array}$ & $\begin{array}{l}V_{L} \\
\mathbf{k m} / \mathbf{s}\end{array}$ & $\begin{array}{l}\text { W } \\
\text { de } \\
\text { g }\end{array}$ & $\begin{array}{l}\text { AR flr } \\
\text { Loc }\end{array}$ & $\begin{array}{l}\text { BE AE } \\
\text { AB deg }\end{array}$ \\
\hline \multirow[t]{3}{*}{$07 / 01 / 25$} & $06: 47$ & $25-40$ & 0.083 & $23: 30$ & DG & 1367 & 36 & 0940 C6.3 & 0.16 \\
\hline & $-06: 50$ & & & & & & 0 & S08E90 & 0.39 \\
\hline & & & & & & & & & 0.55 \\
\hline \multirow[t]{3}{*}{$07 / 02 / 19$} & $00: 18-$ & $30-45$ & 0.2 & $\mathbf{N}$ & 186 & DG & DG & 0943 C1.1 & 0.12 \\
\hline & $00: 21$ & & & & & & & S12E16 & 0.80 \\
\hline & & & & & & & & & 0.92 \\
\hline \multirow[t]{2}{*}{ 07/05/19 } & $12: 51-$ & $38-$ & 0.25 & $13: 05$ & 1624 & 958 & 10 & 0956 B9.5 & 2.15 .9 \\
\hline & $13: 18$ & $167 ?$ & & & & & 6 & N07W06 & 8.0 \\
\hline \multirow[t]{2}{*}{$07 / 05 / 22$} & $14: 36-$ & $25-82 ?$ & 0.05 & $\mathbf{N}$ & 356 & 544 & 10 & 0956 B3.9 & 3.16 .0 \\
\hline & $14: 55$ & & & & & & 8 & N02W42* & 9.1 \\
\hline \multirow[t]{2}{*}{$07 / 05 / 23$} & $07: 22-$ & $25-180$ & 0.07 & $\mathbf{N}$ & 609 & 679 & 90 & 0956 B5.3 & 3.26 .0 \\
\hline & $07: 33$ & & & & & & & N02W55* & 9.2 \\
\hline \multirow[t]{2}{*}{ 07/06/03 } & 09:28- & $28-84$ & 0.15 & $09: 54$ & 491 & 467 & 71 & 0960 C5.3 & 3.97 .1 \\
\hline & $09: 42$ & & & & & & & S08E67 & 11.0 \\
\hline \multirow[t]{2}{*}{ 07/08/06 } & 09:16- & $25-94 ?$ & 0.08 & $\mathbf{N}$ & 333 & 379 & 66 & 966 C1.5 & 9.813 .5 \\
\hline & 09:21 & & & & & & & S05E41 & 23.3 \\
\hline \multirow[t]{3}{*}{$07 / 12 / 31$} & $00: 53-$ & $30-$ & 0.11 & $01: 23$ & 771 & 995 & 16 & 0980 C8.3 & 22.8 \\
\hline & 01:11 & $118 ?$ & & & & & 4 & S08E81 & 21.2 \\
\hline & & & & & & & & & 44.0 \\
\hline \multirow[t]{3}{*}{$08 / 03 / 25$} & $18: 52-$ & $25-60$ & 0.07 & $19: 20$ & 773 & 1103 & 90 & 0989 M1.7 & 23.7 \\
\hline & $19: 00$ & & & & & & & S13E78 & 23.5 \\
\hline & & & & & & & & & 47.2 \\
\hline \multirow[t]{3}{*}{$08 / 04 / 26$} & $14: 12-$ & $25-45 ?$ & 0.024 & $14: 15$ & 335 & 515 & 28 & ?? $\quad$ B3.8 & 24.0 \\
\hline & $14: 22$ & & & & & & 1 & N08E09 & 25.6 \\
\hline & & & & & & & & & 49.6 \\
\hline
\end{tabular}

5 from the SOHO/LASCO CME catalog (cdaw.gsfc.nasa.gov) and is provided to

6 make comparison with other studies of type II bursts in the pre-STEREO era.

7 There were data gaps (DG) during only two of the 10 type II bursts: there was no

8 STEREO data except for a single EUVI and COR1 image for the 2007 January 25 
1 event. The 2007 February type II occurred during a LASCO data gap. Fortunately,

2 information is available in both cases from other coronagraphs. Details of the

3 source region of the eruption such as the NOAA active region number, the GOES

4 soft X-ray flare size, and the heliographic coordinates of the flare are given

5 column 9. For example, the type II burst and CME on 2007 January 25 occurred

6 in AR 0940 accompanied by a C6.3 flare from the location S08E90. An asterisk

7 indicates that the source location was obtained from the previous location by

8 applying solar rotation. The last column (10) gives the separation (in degrees) of

9 STEREO Ahead (SA) and STEREO Behind (SB) with respect to Earth (E) and

10 the separation between SA and SB. For example, at the time of the last event in

11 Table 2 (2008 April 26 type II), SB was $24^{\circ}$ behind Earth (BE) and SA was $25^{\circ} .6$

12 ahead of Earth (AE), while SA and SB were separated by $49^{\circ} .6$ (AB). The last

13 column in Table 2 shows that the separation between the two STEREO spacecraft

14 ranged from $\sim 0^{\circ} .5$ to $\sim 50^{\circ}$. The separation was large enough for the last few

15 events to see clear differences in the appearances of CMEs in the two views.

$17 \quad 2.1$ Statistical Results

18 Some important results can be derived from the information compiled in Table 2.

19 First, even though the solar activity is approaching its minimum (see Fig.1), type

20 II bursts are still produced in all wavelength domains: purely metric, metric to $\mathrm{DH}$

21 (m-DH) and metric to kilometric (m-km). The average speed of the CMEs within

22 the LASCO FOV is $779 \mathrm{~km} / \mathrm{s}$, considerably larger than that of the general

23 population of CMEs ( $\sim 480 \mathrm{~km} / \mathrm{s}$ - see Gopalswamy, 2006a). The width of the

24 CMEs within the LASCO FOV is also $\geq 66^{\circ}$. Such speeds and widths are

25 consistent with the fact that CMEs associated with type II bursts are generally

26 more energetic. CMEs associated with purely metric type II bursts have an

27 average speed of $534 \mathrm{~km} / \mathrm{s}$. On the other hand the five m-DH type II bursts are

28 associated with CMEs with a higher average speed $(808 \mathrm{~km} / \mathrm{s})$. Finally, the single

29 m-km type II burst on 2007 January 25 was associated with the fastest CME in

30 Table $2(1367 \mathrm{~km} / \mathrm{s})$. Despite the small sample, the type II bursts are consistent

31 with the hierarchical relationship between CME kinetic energy and the

32 wavelength range over which the type II bursts occur (Gopalswamy et al., 2005;

33 Gopalswamy, 2006b; 2008a,b). 
1 Second, the flare sizes are generally low: $4 \mathrm{~B}-, 5 \mathrm{C}$ - and a single M-class flares.

2 Thus, 9 out of the 10 flares (or 90\%) were of B and C class. This is in stark

3 contrast to the energetic CMEs involved in the events. Such weak flares are

4 problematic to the non-CME shock origin because the flare thermal energy is

5 typically an order of magnitude smaller than the CME kinetic energy.

7 Third, there is a clear increase in CME speed between the inner and outer corona

8 as measured in SECCHI/COR1 and LASCO FOVs. This is an indication that the

9 CME is accelerating at the time of the metric type II burst. This result has

10 important implications for the behavior of the drift rate spectrum of type II bursts

11 (Gopalswamy, 2006b). The exceptions are the two impulsive events, in which the

12 speed started declining around the time of the metric type II burst. Considering

13 the fact that CMEs liftoff from rest, these two events indicate that the CMEs

14 reached peak speed even before the start of the type II bursts. A detailed analysis

15 of the speed variation will be performed for several events in the next section.

\section{3. Height-time Analyses}

18 One of the main objectives of this study is to determine the CME distance at the

19 time of the type II burst onset. Six of the 10 metric type II bursts had CME

20 observation when the burst was in progress, thus allowing us to obtain the height

21 of the CME leading edge at the time of the burst onset accurately. In this section

22 we describe a few such events with height - time plots and CME images. In

23 particular, we consider the limb events because they are not subject to projection

24 effects. The height - time analysis includes data from STEREO and SOHO. In

25 STEREO, we use the SECCHI/EUVI images to track the earliest disturbances

26 associated with the eruption. These are the disturbances that surround the rising

27 CME, which could be EUV waves or shocks depending on the CME speed. The

28 SECCHI/COR1 images provide CME height - time information in the crucial

29 region where metric type II bursts originate. In the outer corona, CMEs are

30 imaged using SECCHI/COR2 and SOHO/LASCO. Data from SA and SB are

31 distinguished. Thus, there are generally three sets of data points (SA, SB, and

32 SOHO) for each CME. When the spacecraft separation was not large, all the three

33 sets yield nearly identical results. At large separations, the three data sets give 
1 results consistent with the projection effects. In addition, one can also discern

2 differences arising from the different fields of view.

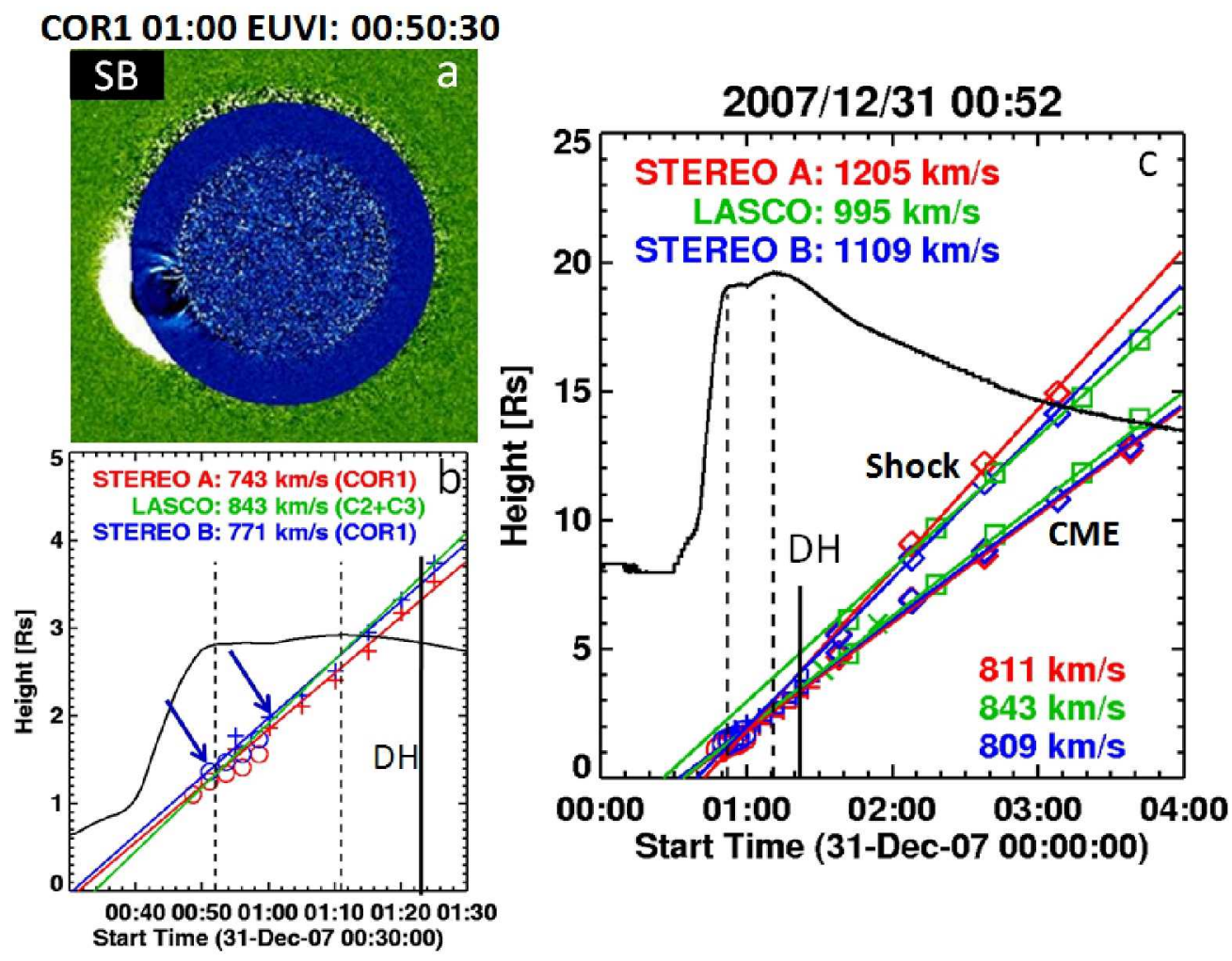

6 Figure 2. (a) CORIB difference image at 01:00 UT superposed on the EUVI 171

7 A difference image at 00:50:30 UT showing the 2007 December 31 CME

8 associated with the metric type II burst at 00:52 UT. The blue annulus is the

9 occulting disk seen above the EUVI limb. (b) Height-time plots of the CME from

10 CORI and EUVI data. STEREO Ahead (SA), STEREO Behind (SB), and SOHO

11 data are color coded as red, blue, and green, respectively (applies to both

12 symbols and lines). Thus red and blue "plus" symbols correspond to CORI

13 measurements from $S A$ and $S B$, respectively. Similarly, red (SA) and blue (SB)

14 circles represent EUVI measurements. The green line is the extrapolation of the

15 SOHO/LASCO measurements made outside the COR1 FOV. The blue arrows

16 point to the measurements from the EUVI and CORI images shown in (a). The

17 two vertical dashed lines mark the start and end times of the metric type II radio

18 burst. The vertical solid line marked "DH" denotes the time when the type II

19 burst at DH wavelengths ended (01:23 UT). (c) Height-time plots of the CME

20 extended to include SOHO and STEREO FOVs. Measurements from the outer 
coronagraphs (C3: squares, COR2: diamonds) are distinguished from those of

2 inner coronagraphs (COR1: "plus" symbols, LASCO/C2: crosses). The circles

3 represent EUVI measurements as in (b). The GOES soft X-ray light curve in the 1

$4-8$ A channel is superposed on the height-time plot for reference. The height-

5 time plots corresponding to the main body of the CME and the diffuse feature

6 (Shock) ahead of the CME are distinguished.

\section{3.1 The 2007 December 31 Event}

9 Figure 2 illustrates the height-time analysis using the 2007 December 31 event.

10 Throughout this paper, height means heliocentric distance, not height above the

11 solar surface. The height - time measurements were made at the fastest moving

12 segment of the CME. The CME can be seen above the southeast limb in COR1

13 and EUVI images (Fig. 2a). The EUVI image shows both the loop-like main body

14 of the CME as well as disturbances on the sides. The dark crescent-shaped feature

15 just above the limb is the CME leading edge in the previous frame, seen here as

16 dark because of the running difference.

17 Figures 2b,c show the height-time history of the CME within the COR1 FOV and

18 the entire FOV, respectively. Several height-time measurements were possible

19 within the time interval of the metric type II burst (3 EUVI and four COR1 data

20 points - see Fig. 2b). The EUVI image taken about a minute before the start of the

21 type II burst (see Fig. 2a) gives the overall initial extent of the shock. Note that

22 the type II burst ended at DH wavelengths just before the CME left the COR1

23 FOV. The linear fits to the height - time plots are all close to each other, with an

24 speed of $\sim 800 \mathrm{~km} / \mathrm{s}$. This because the CME originated close to the limb in the

25 view of all the three coronagraphs. As Table 2 shows, the separation between SA

26 and SB is $\sim 44^{\circ}$; SA was $\sim 21^{\circ}$ ahead of Earth, while SB was $\sim 23^{\circ}$ behind. From

27 Earth view, the eruption occurred at S08E81. As viewed from SA and SB, the

28 source longitude is expected to be E102 and E58 respectively. Thus the SA and

29 Earth views of the CME were almost devoid of projection effects. The projection

30 effects were also minimal for the SB view.

31 Figure 2c shows an extra set of lines (marked "Shock") with data points only

32 from the outer coronagraphs. These measurements correspond to the diffuse outer

33 portion of the CME, which is likely to be the leading shock. The diffuse structures

34 became visible when the CME reached a height of $\sim 5$ Rs. Figure $3 b$ shows a 
1 snapshot from SOHO/LASCO, which shows the diffuse structure (pointed by an

2 arrow). Similar diffuse structure was also observed in the COR2 FOV (not

3 shown). Diffuse structures like this have been reported in association with fast and

4 wide CMEs that are radio quiet (Gopalswamy et al., 2008b; Michalek et al.,

5 2007). It is possible that these shocks are weak and start separating from the

6 driving $\mathrm{CME}$ as the CME slows down in the near-Sun IP medium. In the COR1

7 image shown in Fig. 2a, it is difficult to see any diffuse structure at the leading

8 edge, which suggests that the shock is close to the CME leading edge and cannot

9 be seen distinctly. However, such diffuse structure can be seen at the flanks of the

10 CME.
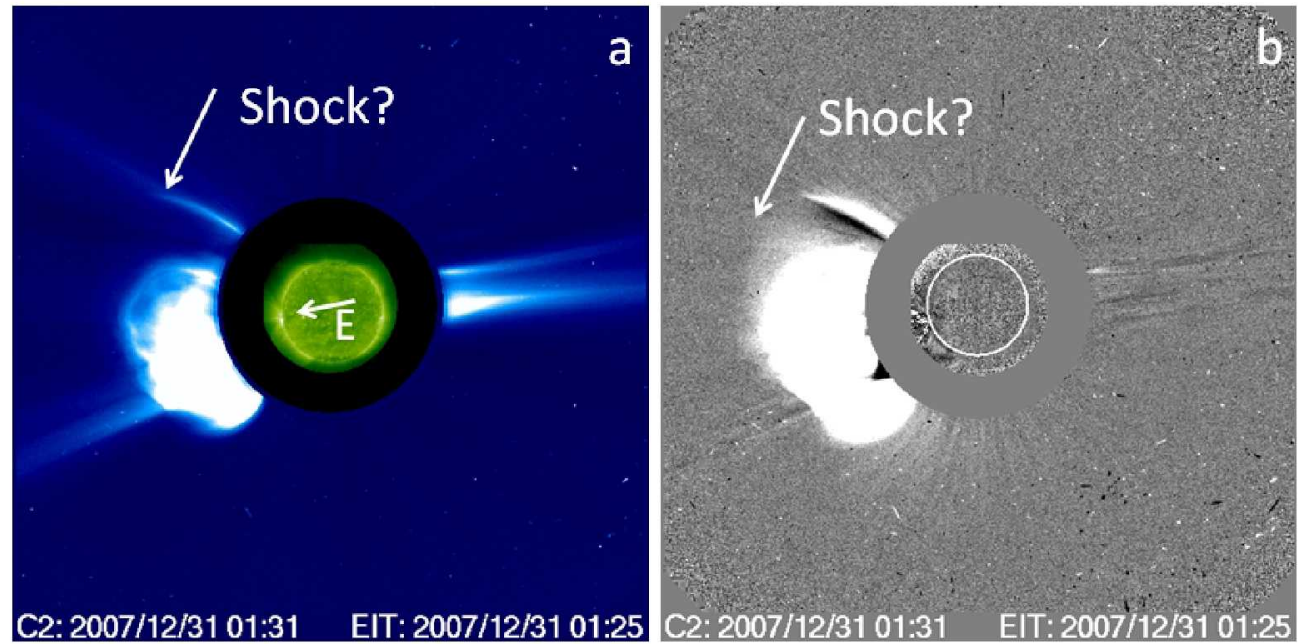

12 Figure 3. (a) LASCO/C2 image of the 2007 December 31 CME at 01:31 UT from

13 the eruption center $(E)$ at the east limb. The kink in the streamer to the north of

14 the CME is indicative of the shock. (b) Difference image of the same CME

15 showing diffuse filling of material up to the "kink". The diffuse structure marked

16 "Shock?" extends from the nose and corresponds to the outermost disturbance.

17 The disturbance can also be seen in the SOHO/EIT difference image superposed

18 on the LASCO image. The LASCO image was taken slightly after the end of the

19 type II burst.

\section{CME Height at Metric Type II Onset}

22 The metric dynamic spectrum is rather complex. The type II starts with a

23 fundamental - harmonic structure at 00:52 UT with the harmonic band showing

24 band-splitting. The fundamental and harmonic components seem to continue into

25 the SWAVES and WAVES frequency range as shown in Fig. 4. The fundamental 
1 component is somewhat weak in the metric domain. Both the fundamental and

2 harmonic components end around 01:23 UT in the SWAVES

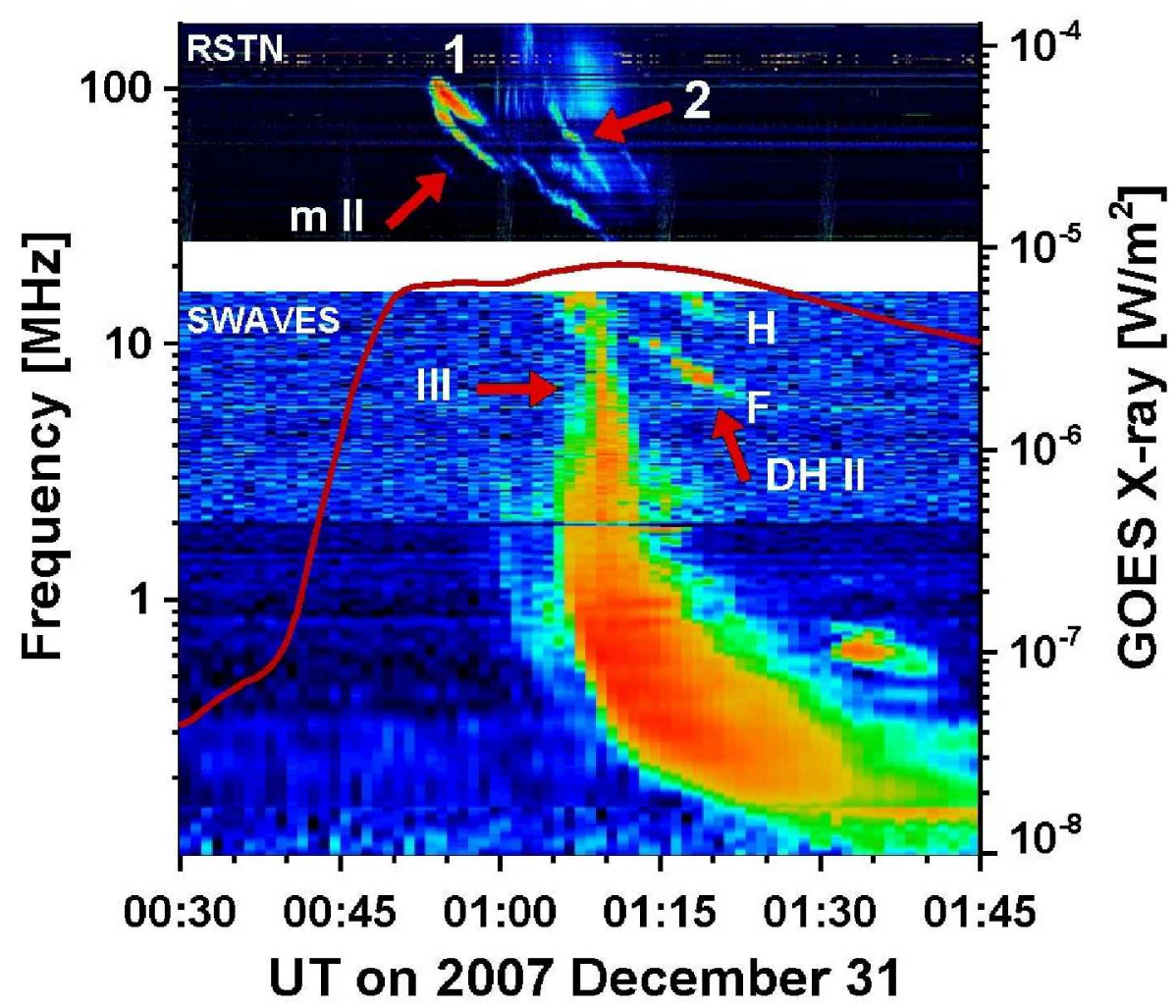

$4 \quad$ Figure 4. Composite dynamic spectrum showing the metric type II burst (m II)

5 observed at the Learmonth Observatory belonging to the Radio Telescope

6 Network (RSTN) and the DH type II burst (DH II) observed by STEREO/WAVES

7 (SWAVES) on 2007 December 31. The GOES time profile shows two peaks.

8 There seems to be two episodes (marked 1 and 2) of the metric type II burs, with

9 the second one being brief with fundamental $(F)$ - harmonic $(H)$ structure. The

10 DH II shows F-H structure, which is a continuation of the first metric type II

11 episode (marked 1). The DH type III burst is marked as "III". The fundamental

12 components of the type II in $m$ and DH domain are indicated by arrows.

14 dynamic spectrum. An additional metric type II burst (marked 2) with a different

15 drift rate seems to be superposed on the first one (see Fig 4 around 01:05 UT).

16 The GOES time profile also shows two peaks, the second one coinciding with the

17 second episode of type II burst. The DH type III bursts also start only during the

18 second episode. Since there is no other eruption from other regions, we think the 
1 two episodes of type II bursts are from the same eruption. It is possible that the

2 shock encounters a dense streamer and produces the second episode. It is also

3 possible that early in the eruption, the flare-accelerated electrons did not have

4 access to the open field lines, hence no type III bursts.

5 The height-time plots in Fig. 2 show that at the time of the metric type II burst

6 onset, the location of the CME leading edge was at 1.32, 1.46, and 1.39 Rs,

7 respectively from the $\mathrm{SA}, \mathrm{SB}$, and $\mathrm{SOHO}$ data. These heights are very close to

8 each other and smaller than the average height of $\sim 2.2$ Rs obtained using LASCO

9 data alone (Gopalswamy et al., 2005). We conclude that the heliocentric distance

10 of the shock is not too different from $\sim 1.46$ Rs. When the DH type II burst ended

11 at 01:23 UT, the CME was at a height of $\sim 3.5$ Rs. This means the shock was able

12 to accelerate electrons to sufficient numbers only over a short distance of $\sim 2$ Rs.

13 In the following, we attempt to explain why this is so.
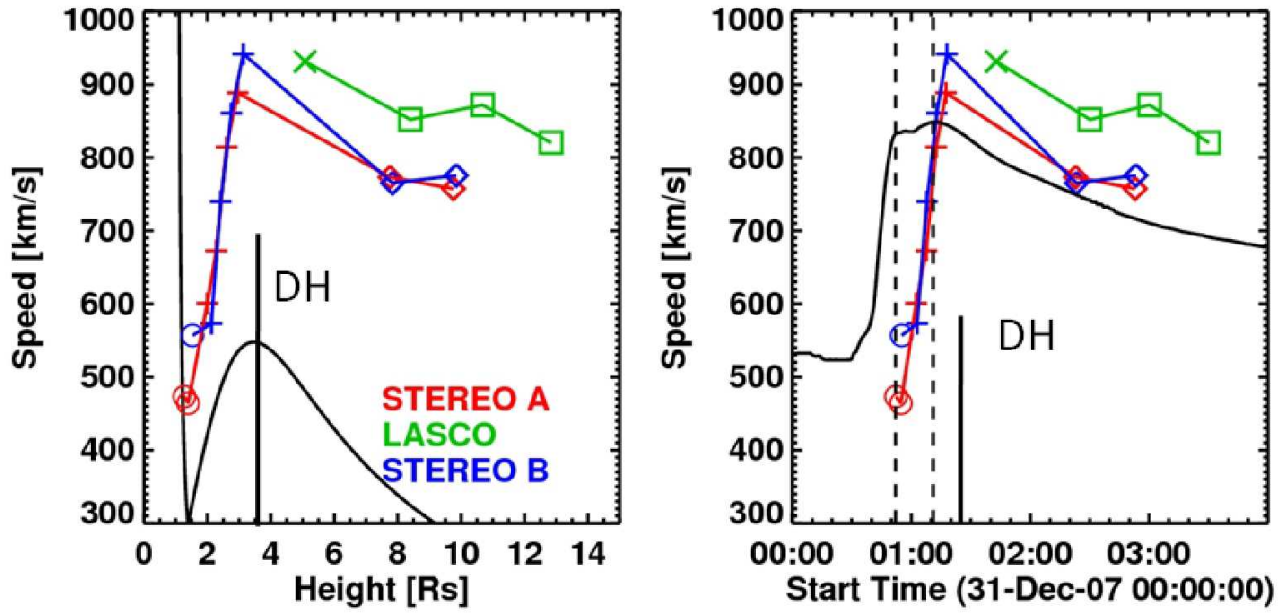

Figure 5. (left) Variation of the CME speed for the 2007 December 31 event as a function of height derived from the height-time plots in Fig. 2. Variation of the Alfven speed as a function of height (dark curve) is superposed on the plot. (right) Variation of the CME speed as a function of time. The GOES soft X-ray light curve is superposed on the speed - time plot. The interval of the metric type II burst is marked by the two vertical dashed lines. The end of the DH type II burst is indicated by the solid vertical line marked " $D H$ ". Note that the type II emission ends immediately after the speed starts decreasing from its maximum value ( 950 $\mathrm{km} / \mathrm{s}$ ). The speed reaches its maximum value when the CME is at a height of $3 \mathrm{Rs}$. The speed profile peaks slightly after the flare peak, which is different from the usual scenario. 


\section{CME Speed Evolution and Cessation of Type II Burst}

2 Since there are sufficient numbers of height-time measurements in each view, we

3 were able to follow the evolution of the CME speed in time. We combined the

4 measurements at various distance ranges in each view (SA, SB, and SOHO).

5 From STEREO data, the first data point is from EUVI, the next four are from

6 COR1 and the last two are from COR2. In LASCO data, the first speed

7 measurement is from $\mathrm{C} 2$ and the rest are from $\mathrm{C} 3$. The speeds were determined by

8 fitting a straight line to four successive height-time data points. Figure 5 shows

9 the CME speed as a function of heliocentric distance and time for the 2007

10 December 31 event. We see that the speed increases from $\sim 475 \mathrm{~km} / \mathrm{s}$ near the

11 start of the type II burst, reaches a maximum value of $\sim 950 \mathrm{~km} / \mathrm{s}$ at $\sim 01: 15$ UT

12 and then declines. The maximum speed reached is slightly different as measured

13 in SA ( $888 \mathrm{~km} / \mathrm{s}$ at $3.0 \mathrm{Rs})$ and SB $(941 \mathrm{~km} / \mathrm{s}$ at $3.1 \mathrm{Rs})$. The difference in peak

14 speeds $(\sim 7 \%)$ is not significant because the position angle at which the CME

15 leading edge was tracked is slightly different for SA and SB. Note that there was

16 no COR1 speed measurement after the peak, so the peak value is only

17 approximate. Judging by the $\mathrm{C} 2$ data point, it is possible that the speed reached a

18 slightly higher value before declining. In the declining part, the speed

19 measurements are available only from COR2 (2 data points) and LASCO (4 data

20 points). LASCO speeds are slightly higher, but only by about $10 \%$.

22 By the time the type II burst ended at 01:23 UT, the CME was still fast with speed

$23>800 \mathrm{~km} / \mathrm{s}$. The speed had declined only to $\sim 800 \mathrm{~km} / \mathrm{s}$ by the time the CME

24 reached the edge of the LASCO/C3 FOV. When the CME was moving with a

25 speed of $\sim 500 \mathrm{~km} / \mathrm{s}$, it was associated with a type II burst, but not when it was

26 moving as fast as $800 \mathrm{~km} / \mathrm{s}$. Why is this contradiction? To understand this, we

27 need to look at how the characteristic speed of the ambient medium

28 (Magnetosonic speed or Alfven speed) changes with height. In the core of active

29 regions, the characteristic speed is high (more than $1000 \mathrm{~km} / \mathrm{s}$ ), which drops

30 rapidly in the outskirts of the active region to $\sim 300 \mathrm{~km} / \mathrm{s}$. The characteristic speed

31 again starts rising, and reaches a maximum around $550 \mathrm{~km} / \mathrm{s}$ (Mann et al., 1999;

32 Gopalswamy et al., 2001a) in the height range 3-4 Rs. In Fig. 5, we have overlaid

33 the radial profile of the Alfven speed (Va) computed using Saito et al. (1977)

34 density model as in Gopalswamy et al. (2001a), except that we have used Va 
1 instead of the magnetosonic speed. The radial evolution of the characteristic speed

2 is somewhat parallel to that of the CME speed except in the core of the active

3 region. Near the start of the Type II, the Alfvenic Mach number is 1.7 (556/329

4 in EUVI-B). When the CME reaches its peak speed, the Mach number remains

5 the same (941/542 in COR1B). It is interesting that the type II burst ends when the

6 CME speed starts declining (see Fig. 5). When the type II ended, the CME speed

7 was somewhere between 850 and $900 \mathrm{~km} / \mathrm{s}$ and the Alfven speed has reached its

8 maximum value $(\sim 550 \mathrm{~km} / \mathrm{s})$, suggesting that the Mach number is in the range 1.5

$9-1.6$. The Mach number is expected to be even lower, when the solar wind

10 speed, which begins to become significant around $3 \mathrm{Rs}$, is taken into account (see,

11 e.g., Sheeley et al., 1997; Gopalswamy et al. 2001a). A shock with such a low

12 Mach number is likely to be a subcritical shock and hence the particle acceleration

13 efficiency is expected to be low (Mann et al., 2003). Consequently, enough

14 number of electrons may not be accelerated to produce the type II burst. The

15 combination of the characteristic speed of the medium and the CME speed seem

16 to be such that the shock either became too weak or decayed by the time the CME

17 reached the outer corona. It is worth noting that the peak Va shown in Fig. 5 is

18 only a representative value, which can be much higher or lower depending on the

19 actual density and magnetic field in the corona. Furthermore, the solar wind speed

20 reaches about $150 \mathrm{~km} / \mathrm{s}$ at $\sim 5 \mathrm{Rs}$ (see Sheeley et al., 1997), so shock-driving gets

21 more difficult because the solar wind speed needs to be subtracted from the CME

22 speed before comparing with Va to get the Mach number. Thus, CME speed

23 decrease and solar wind speed increase weaken the shock, while the decreasing

24 Alfven speed makes the shock stronger. The combination of the three speeds

25 decides the existence and the strength of the shock.

$26 \quad 3.2$ The 2008 March 25 Event

27 In many ways the 2008 March 25 event is similar to the 2007 December 31 event:

28 it is an east-limb CME with an m-DH type II radio burst and the shock component

29 was discernible after the end of the DH type II burst. Even the morphology of the

30 CME in EUVI and COR1 images is nearly identical to that of the 2007 December

$3131 \mathrm{CME}$. The EUVI $171 \AA$ image in Fig. 6a shows the curved-front CME with

32 extended disturbances near the limb on the northern and southern edges and on

33 the disk. This is the EUV disturbance that surrounds the CME. The image in Fig.

34 6a was obtained only a minute after the start of the type II burst and demonstrates 
1 that the shocks can form very close to the Sun. The leading edge of the EUVI

2 CME is at a height of 1.47 Rs. In the minute before the start of the type II, the

3 EUVI CME was at a height of 1.3 Rs. Thus the CME was somewhere between 1.3

4 and 1.47 Rs when the type II burst started. The COR1 image shown in Fig. 6a was

5 taken $\sim 1.5$ min after the superposed EUVI image, so it is slightly larger in size.

6 The height-time plots in Fig. 6 give a CME leading edge height of 1.42 Rs,

7 consistent with the EUVI observation. From Earth view, the CME occurred at

$8 \mathrm{~S} 13 \mathrm{E} 78$, so it is slightly on the disk in the SB view because SB was $\sim 24^{\circ}$ behind

9 Earth at the time of the eruption. Similarly, the CME was slightly behind the limb

10 for SA, which was $\sim 24^{\circ}$ ahead of Earth, so the CME leading edge at the time of

11 the type II was at 1.56 Rs. The nearest SA image in EUVI was at 18:51 UT, one

12 minute before the type II onset and showed the CME leading edge to be at 1.50

13 Rs. Thus, a height of $\sim 1.5$ Rs for the CME leading edge at the time of type II burst

14 onset is a good estimate. The SOHO/LASCO observations give a leading edge

15 height of 1.65 Rs at the type II onset, which is substantially higher than the

16 STEREO values. This is mainly because the average speed in the LASCO FOV is

17 relatively higher $(1037 \mathrm{~km} / \mathrm{s}$ - see Fig. $6 \mathrm{~b})$. At first appearance (at 19:31 UT - see

18 Fig. 7) in LASCO FOV, the CME was already at a height of $5.79 \mathrm{Rs}$, requiring

19 too much extrapolation to the time of type II onset. As we showed for the 2007

20 December 31 event, the CME speed varies significantly between the CME lift off

21 and its appearance in the LASCO FOV, which is not captured in LASCO

22 observations.

COR1 18:55 EUVI: 18:53:53
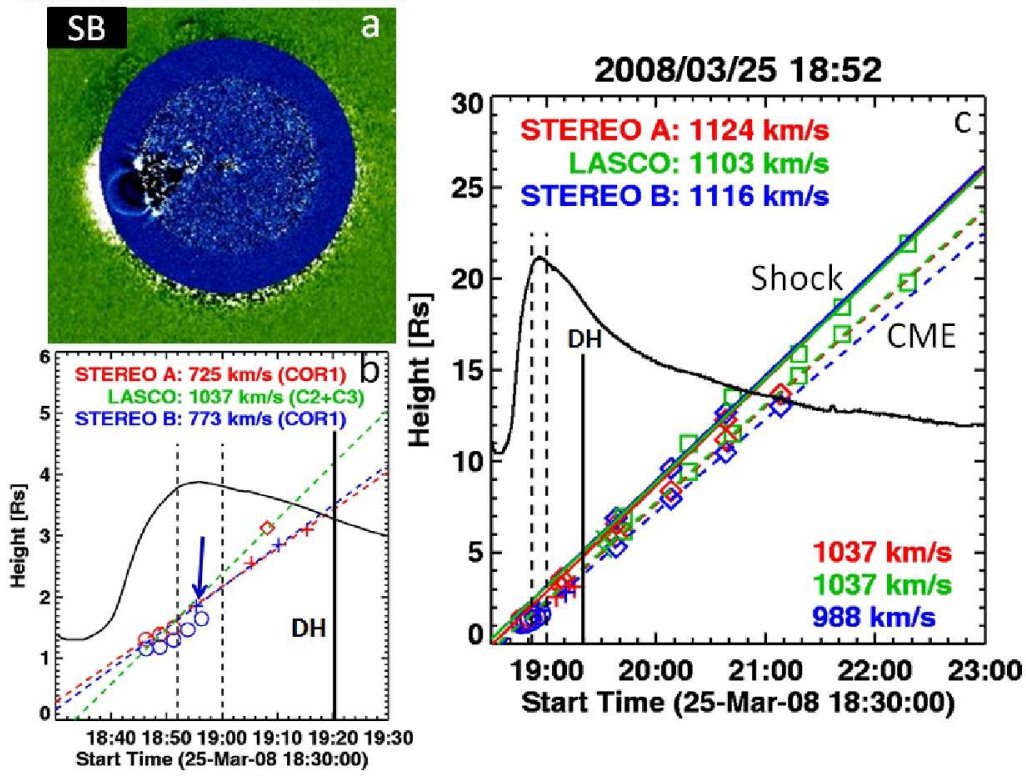
1 Figure 6. (a) COR1B difference image at 18:55:23 UT superposed on the EUVI

2171 A difference image at 18:53:53 UT showing the 2008 March 25 CME when

3 the metric type II burst was in progress. (b) CME height-time plot within the

4 CORI FOV with EUVI and CORI data points. The symbols have the same

5 meaning as in Fig. 2. The arrow points to the COR1 and EUVI measurements

6 from the images shown in (a). The two vertical dashed lines mark the start and

7 end times of the metric type II radio burst. The vertical line marked " $D H$ "

8 denotes the time when the type II burst at DH wavelengths ended (19:20 UT). (c)

9 Extended height-time plots from SOHO and STEREO, with the symbols and colors

10 having the same meaning as in Fig. 2. The GOES soft X-ray light curve in the $1-$

118 A channel is superposed on the height-time plot for reference. The height-time

12 plots in dashed and solid lines correspond to the main body of the CME and the

13 shock as labeled.
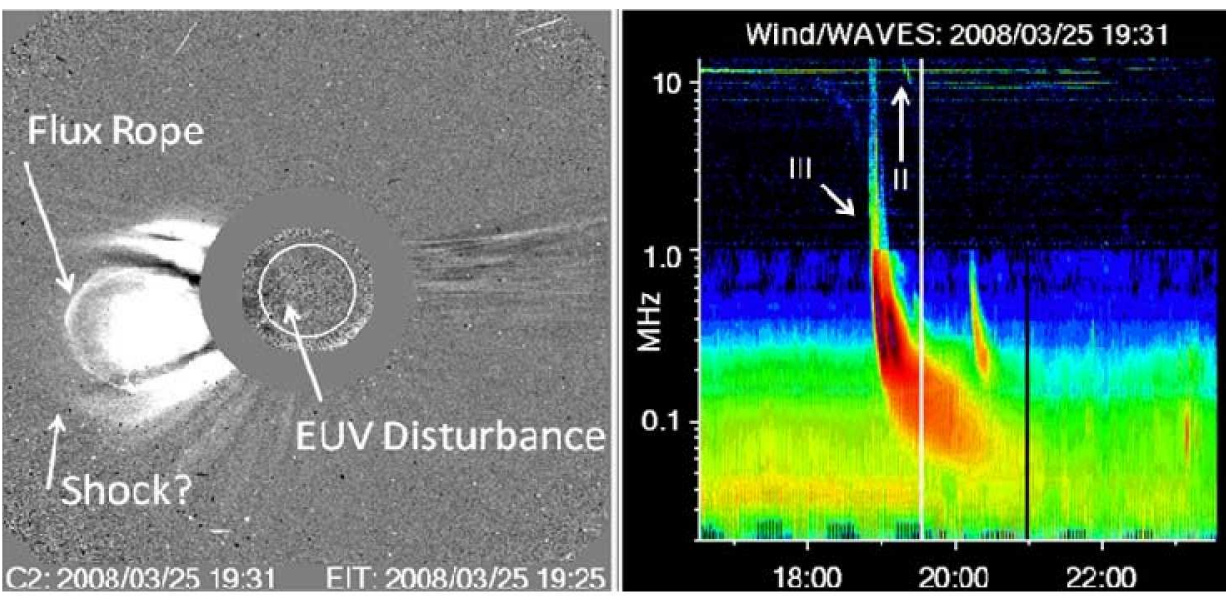

16 Figure 7. (left) The 2008 March 25 CME when it first appeared in the LASCO/C2

17 FOV. The CME had a flux rope structure (marked) and a leading diffuse feature,

18 whose outermost edge we interpret as the shock. The EUV disturbance seen above

19 the limb and on the disk coincides with the outermost part of the white light

20 disturbance that surrounds the flux rope. (right) Wind/WAVES dynamic spectrum

21 with the type III and type II bursts marked. The vertical line marks the time (19:31

22 UT) of the LASCO frame on the left.

24 The Wind/WAVES dynamic spectrum for the 2008 March 25 event is shown in

25 Fig. 7 along with the LASCO image when the CME first appeared. An EUV

26 difference image obtained by SOHO/EIT at 19:25 UT is also shown superposed 
1 on the LASCO image. The extent of the EUV disturbance closely matches the

2 outermost disturbance in white light. Such comparisons were previously made by

3 Thompson et al. (2000), who concluded that the extended dimming areas mapped

4 out the apparent "foot-print" of the CME. Here we note that the CME can be

5 seen as a distinct flux rope structure and the outermost white-light disturbance can

6 be traced above the flux rope as a diffuse feature, which is likely to be the sheath

7 of the CME-driven shock. The flux-rope structure, the outermost sharp feature in

8 Fig. 7, is clearly confined to the central portion of the EUV disturbance

9 suggesting that the flux rope is the driver of the EUV wave. As in the case of the

102007 December 31 event, the shock was not seen distinctly ahead of the COR1

11 CME (but disturbance was seen at the sides low in the corona). The leading

12 diffuse feature can be seen only in the outer corona, that too after the end of the

13 DH type II burst. This suggests that the shock is getting decoupled from the CME

14 because it gets weaker. Note that the DH type II burst ends at 19:20 UT, which is

15 even before the CME appeared in the LASCO FOV. The lowest frequency of the

16 type II burst which appears as the continuation of the metric type II burst was $\sim 10$

17 MHz. From the height-time plot in Fig. $6 \mathrm{~b}$ we see that the CME leading edge was

18 at a height of $\sim 3.6$ - 3.7 Rs when the type II burst ended at $10 \mathrm{MHz}$.
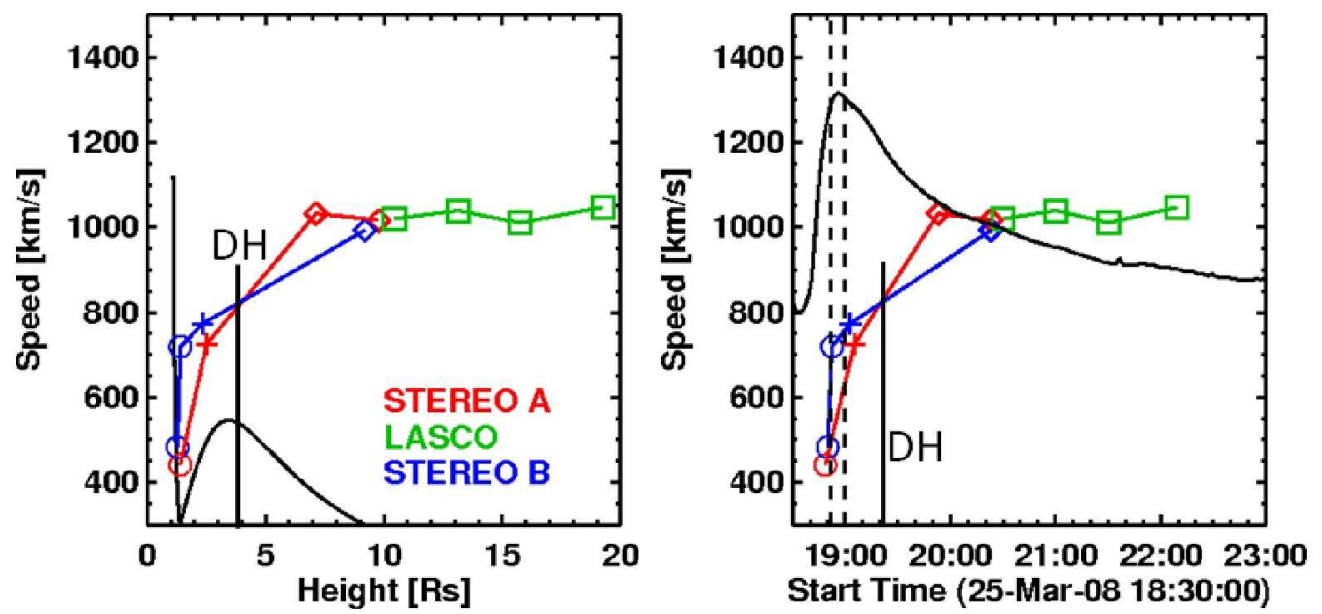

20 Figure 8. Variation of the CME speed with height (left) and time (right) for the

212008 March 25 CME. The duration of the metric type II burst (vertical dashed

22 lines) and the end of the DH type II burst (solid vertical line) are marked. The

23 Alfven speed profile is overlaid on the speed - height plot as in Fig. 5.

25 Figure 8 shows that the CME speed increases rapidly when the metric type II

26 burst is in progress. Unfortunately, there is no CME observation between the end 
1 of the metric type II and the end of the DH type II. But the shape of the speed

2 profile suggests that the speed should have peaked somewhere before the end of

3 the DH type II. Since the speed profile is expected to be similar to that of the

4 GOES soft X-ray profile (Zhang et al., 2001), it is likely that the CME speed

5 peaked before the end of the DH type II. The end of the DH type II coincides with

6 the distance at which Va peaks, very similar to what happened in the 2007

7 December 31 event. The CME speed attained a constant value $(\sim 1000 \mathrm{~km} / \mathrm{s}$ in

8 SA, SB, and SOHO) after the end of the type II burst. This speed is higher than

9 that when the metric type II was in progress $(\sim 800 \mathrm{~km} / \mathrm{s})$. This means the peak

10 CME speed must have been above $1000 \mathrm{~km} / \mathrm{s}$. The lack of type II burst beyond

11 19:20 UT even with a CME speed of $\sim 1000 \mathrm{~km} / \mathrm{s}$ suggests that the Alfven speed

12 in the ambient medium is close to $1000 \mathrm{~km} / \mathrm{s}$ or only slightly smaller, making the

13 shock too weak to produce the type II burst. The tenuous-looking corona between

14 the two streamers (see Fig. 7) into which the CME was launched may have had a

15 higher characteristic speed than the $550 \mathrm{~km} / \mathrm{s}$ indicated by the Va profile in Fig. 8 .

16 The metric type II burst itself might have come from the flanks of the CME,

17 where the streamers are located. When the shock traverses through the streamers,

18 it encounters lower Alfven speed and higher Mach number. Once the shock

19 crosses the streamer region in the lateral direction, the shock enters into a higher

20 Alfven speed region, rendering the shock very weak and the consequent end of the

21 metric type II burst. In summary, the 2008 March 25 event is consistent with the

22 interpretation provided for the better observed 2007 December 31 event.

\subsection{The 2007 June 3 Event}

25 The metric type II burst on 2007 June 3 was associated with an east limb CME

26 and a C5.3 flare from AR 0960 located at S08E67. The separation between SA

27 and SB was only $\sim 11^{\circ}$, so the eruption was a limb event for all the three views.

28 Accordingly, the CME appeared nearly the same as viewed by STEREO and

29 SOHO (see Fig. 9 for nearly identical height-time plots). The metric type II burst

30 lasted for $\sim 14 \mathrm{~min}$, starting at 09:28 UT and ending at 09:42 UT. The

31 Wind/WAVES dynamic spectrum showed a faint and thin slanted feature, which

32 seems to be a continuation of the harmonic component of the metric type II burst.

33 The DH component continued down to $\sim 3 \mathrm{MHz}$ and ended at 09:54 UT. There

34 was only one COR1 image each from SA and SB during the metric type II burst, 
1 but there were several images outside the type II interval. Figure 9a shows a

2 EUVI - COR1 composite image from SA showing the CME at a height of 1.87 Rs

3 and overlying the eruption in EUV. The corresponding CME in SB was at a

4 height of 1.83 Rs (09:36 UT). The height-time plots give the CME height as 1.66,

5 1.6, and 1.65 Rs as obtained from SA, SB and SOHO, respectively. The eruption

6 is closest to the limb in the view from SA, so we can say that the CME leading

7 edge was at a height of $\sim 1.66$ Rs when the type II burst started. The GOES soft

8 X-ray plot superposed on the height-time plot shows that the flare was extremely

9 impulsive, lasting only $\sim 8 \mathrm{~min}$. There were two EUVI images of the eruption well

10 before the start of the type II burst. When the speed was computed as a function

11 of time, we found something remarkable: the CME speed had already reached its

12 peak when the type II burst started (see Fig. 10). The peak speed also coincided

13 with the peak of the soft X-ray flare. The peak CME speed was $\sim 925 \mathrm{~km} / \mathrm{s}$ (SA)

14 near the start of the metric type II burst and dropped to $\sim 500 \mathrm{~km} / \mathrm{s}$ when the type

15 II burst ended at 09:54 UT in the DH domain. The speed evolution closely

16 followed the impulsive soft X-ray profile and dropped below the local Va when

17 the type II burst ended. In this case, the shock itself should have disappeared

18 whereas in the previous cases, the shocks weakened and became subcritical.

19 Contrary to the previous events, the type II burst ended even before Va reached its

20 peak value. The CME first appears in the LASCO/C2 FOV with its leading edge

21 at $2.6 \mathrm{Rs}$ when the type II burst just ended at $3 \mathrm{MHz}$. Thus, the formation and

22 disappearance of the shock were completed even before the CME appeared in the

23 LASCO FOV. 

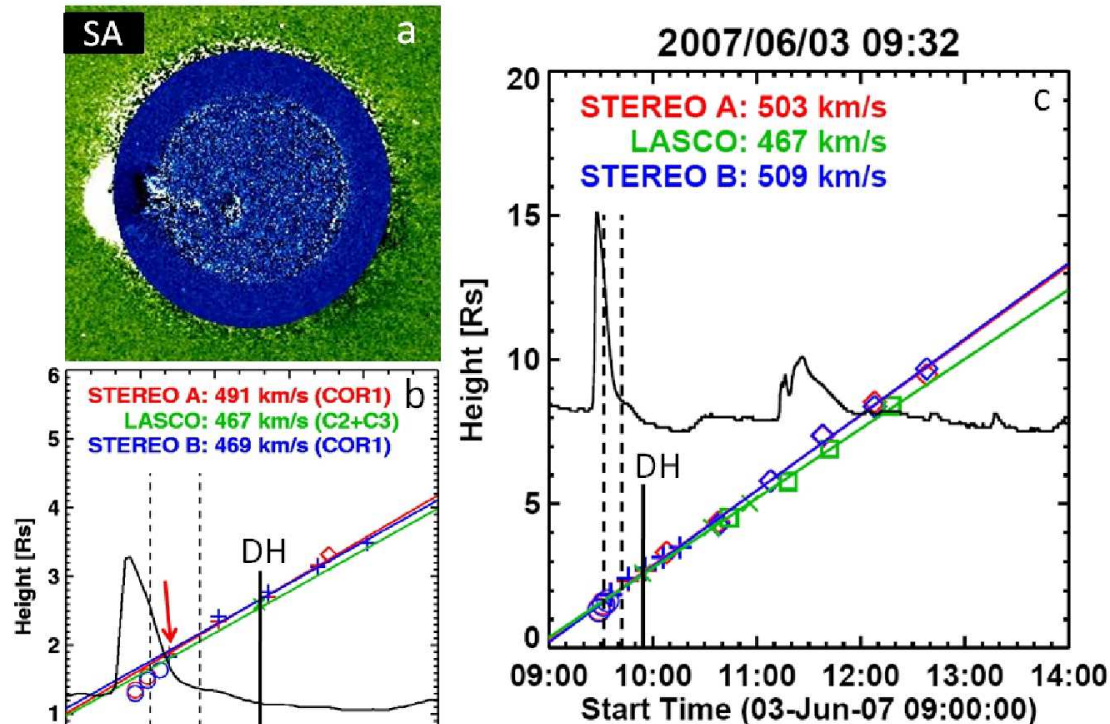

2 Figure 9. (a) COR1A difference image at 09:35:18 UT superposed on the EUVI

3171 A difference image at 09:33:30 UT showing the 2007 June 3 CME when the

4 metric type II burst was in progress. (b) Height-time plots of the CME within the

5 CORI FOV. The arrow points to the data point corresponding to the COR1 image

6 in (a). Times of metric type II (between the dashed lines) and the end of DH type

7 (vertical solid line at 09:54 UT) are marked. (c) CME height-time plots from

8 SOHO and STEREO, with the symbols and color having the same meaning as in

9 Fig. 2. The GOES soft X-ray light curve in the 1-8 A channel is superposed on

10 the height-time plot for reference. The metric type II duration and the end time of

11 DG type II are shown as in (b).
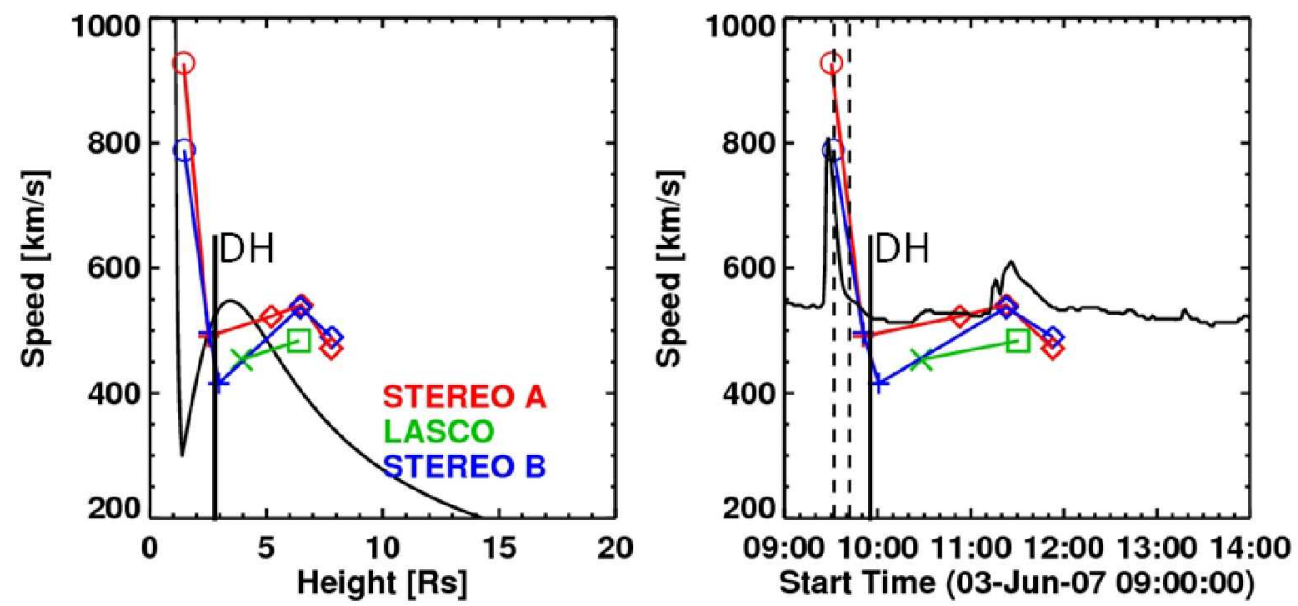

Figure 10. CME speed as a function of height (left) and time (right) for the 2007

14 June 3 event. The GOES soft $X$-ray light curve and the duration of the metric of 
1 the type II burst (two vertical dashed lines) are also shown. The vertical solid line

2 marks the end of the DH type II burst. The Alfven speed profile is shown in the left

3 panel.

\section{$5 \quad 3.42007$ January 25 Event}

6 The 2007 January 25 CME is the only event associated with $\mathrm{m}-\mathrm{km}$ type II burst.

7 There was no metric type II burst reported in the Solar Geophysical Data, but

8 when we examined the dynamic spectrum available at the National Geophysical

9 Data Center (NGDC), we found a weak type II feature in the interval 06:47 UT to

$10 \quad 06: 49 \mathrm{UT}$ drifting from $40 \mathrm{MHz}$ to $25 \mathrm{MHz}$. The drift rate was $\sim 0.1 \mathrm{MHz} / \mathrm{s}$,

11 which is typical of metric type II bursts (Mann et al., 1996). STEREO data

12 coverage was very poor for this event with no SA observations and only one

13 COR1 image in SB at 06:53 UT (see Fig. 11a), which was taken after the metric

14 type II burst. The EUVI image superposed on the COR1 image shows the EUV

15 disturbance extending to the outskirts of the COR1 CME at the limb. The CME

16 leading edge from COR1 (plus symbol on the height - time plot) is consistent

17 with the first appearance time of the CME in LASCO/C2 FOV with its leading

18 edge at $\sim 2.98$ Rs. The CME height-time plot is mainly from LASCO/C3 images

19 with just single data point from COR1B and LASCO/C2 (see Fig. 11). The CME

20 speed was obtained only from the LASCO data for this event. The high speed of

21 the CME $(1367 \mathrm{~km} / \mathrm{s})$ is consistent with the expected speed for an m-km type II

22 burst (the average speed of all m-km type II bursts is $\sim 1500 \mathrm{~km} / \mathrm{s}-$ see

23 Gopalswamy et al., 2005). The speed variation shown in Fig. 11c is also

24 consistent with the m-km type II burst because the CME speed is much larger than

25 the Alfven speed and hence must be driving a shock throughout the inner

26 heliosphere.
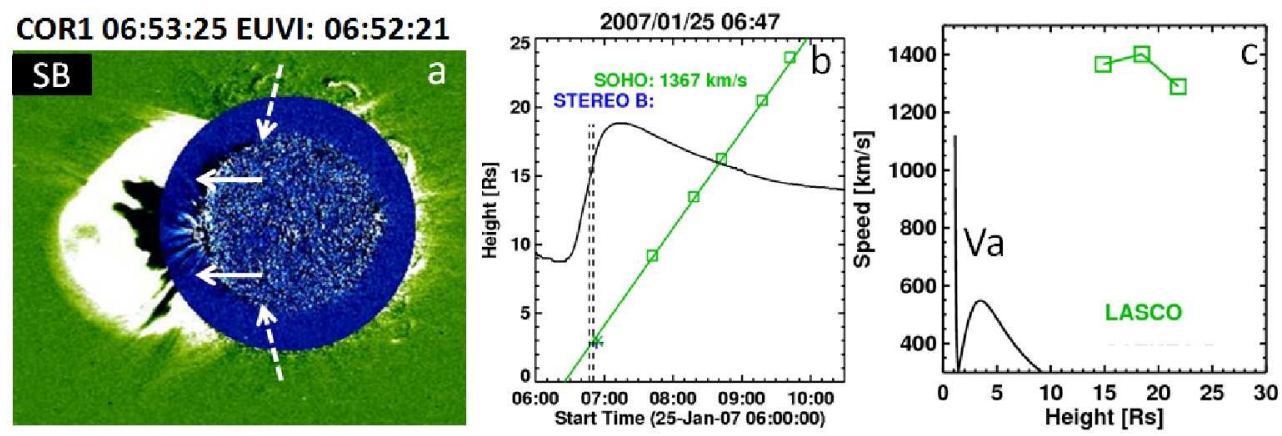
1 Figure 11. (a) COR1 image from SB showing the 2007 January 25 CME during

2 its early phase (at 06:53UT). The leading edge of the CORI CME is at 2.85 Rs.

3 The solid arrows point to the main body of the CME. The dashed arrows point to

4 the outer edge of the EUV disturbance, coinciding with the outermost part of the

5 CME-associated disturbance in white light. (b) CME height-time plot from

6 SOHO/LASCO with the single data points from CORI (plus symbol,

7 corresponding to the image on the left) and LASC/C2 (cross symbol). The GOES

8 soft X-ray light curve and the interval of metric type II burst (shown by two

9 vertical dashed lines) are over-plotted for reference. (c) CME speed variation as

10 a function of heliocentric distance with superposed Alfven speed profile (Va).

12 The m-km type II burst drifted from $14 \mathrm{MHz}$ and reached $\sim 90 \mathrm{kHz}$ by the end of 13 the day (23:30 UT). In LASCO, the CME was tracked from 2.98 Rs at 06:54 UT

14 (when the CME first appeared in the LASCO FOV) to 27 Rs at 10:20 UT (when

15 the CME left the LASCO FOV). The corresponding frequencies are $\sim 14 \mathrm{MHz}$ to

$16 \sim 0.5 \mathrm{MHz}$. Figure 12 shows the LASCO/C3 image of the CME at 08:18 UT, and

17 the Wind/WAVES dynamic spectrum. At this time, the CME leading edge had

18 reached a heliocentric distance of $\sim 13.5$ Rs with the radio emission occurring at a

19 frequency of $\sim 0.9 \mathrm{MHz}$. Unlike the 2007 December 31 and 2008 March 25

20 events, we do not see the shock-like structure at the leading edge of the CME.

21 This may be because the shock may still be close to the CME. However, we do

22 see diffuse structure at the flanks (see Fig. 12).
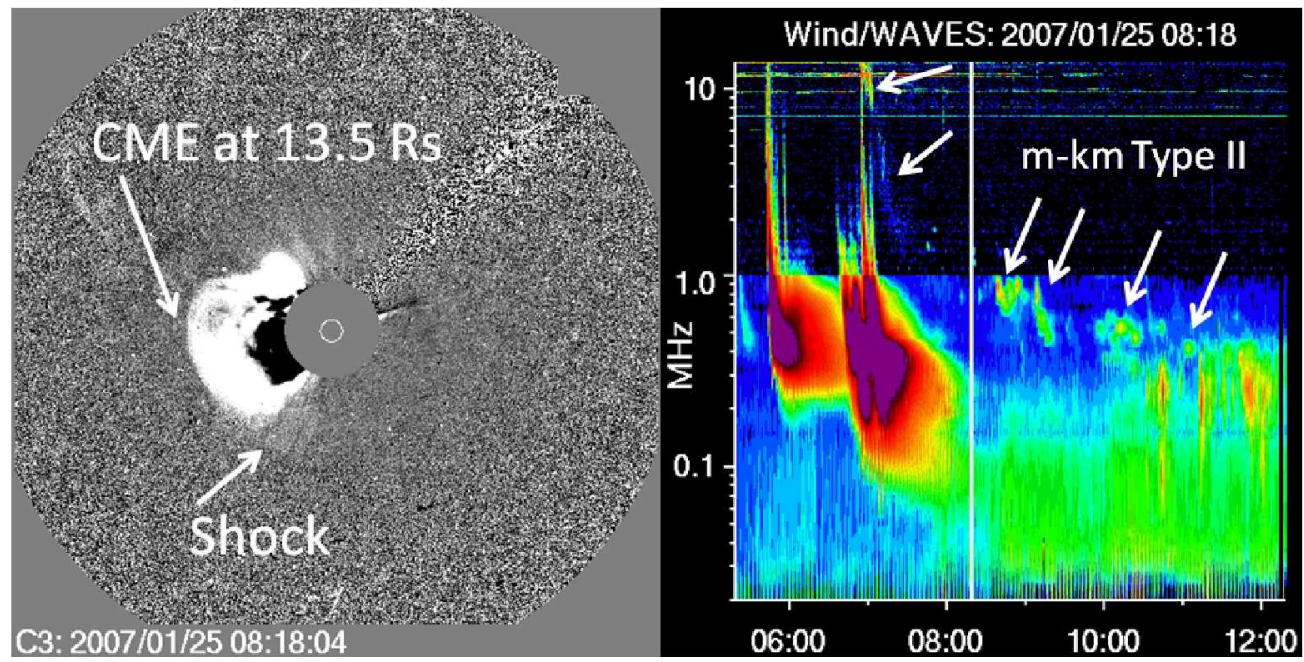

24 Figure 12. Wind/WAVES dynamic spectrum showing the $\mathrm{m}-\mathrm{km}$ type II burst

25 (right) and a snapshot (08:18 UT) of the associated CME from SOHO/LASCO 
1 (left). The type II burst is fragmented as indicated by the arrows. It is faint above

$21 \mathrm{MHz}$ because of the lower sensitivity of the WAVES instrument in this frequency

3 range. The vertical white line marks the time (08:18UT) of the LASCO frame on

4 the left, which shows the frequency of the type II burst $(\sim 0.9 \mathrm{MHz})$ corresponding

5 to the CME height $(13.5 \mathrm{Rs})$. The faint shock-like feature is marked on the CME

6 image.

8 The significant overlap between the LASCO FOV and the plasma levels

9 corresponding to the radio emission can be exploited to understand the relation

10 between CME evolution and the type II burst in the IP medium. Since the shock

11 is not seen distinctly from the CME leading edge, we assume that the CME height

12 is approximately the same as the shock height. With this assumption, we see that

13 the radio emission occurring at a frequency of $\sim 500 \mathrm{kHz}$ must come from a

14 heliocentric distance of $\sim 27$ Rs. While there is no CME observation beyond 32

15 Rs, the radio emission continued to lower frequencies, which means the shock

16 remained strong for another half a day or so when the radio emission reached 90

$17 \mathrm{kHz}$.

Wind/WAVES

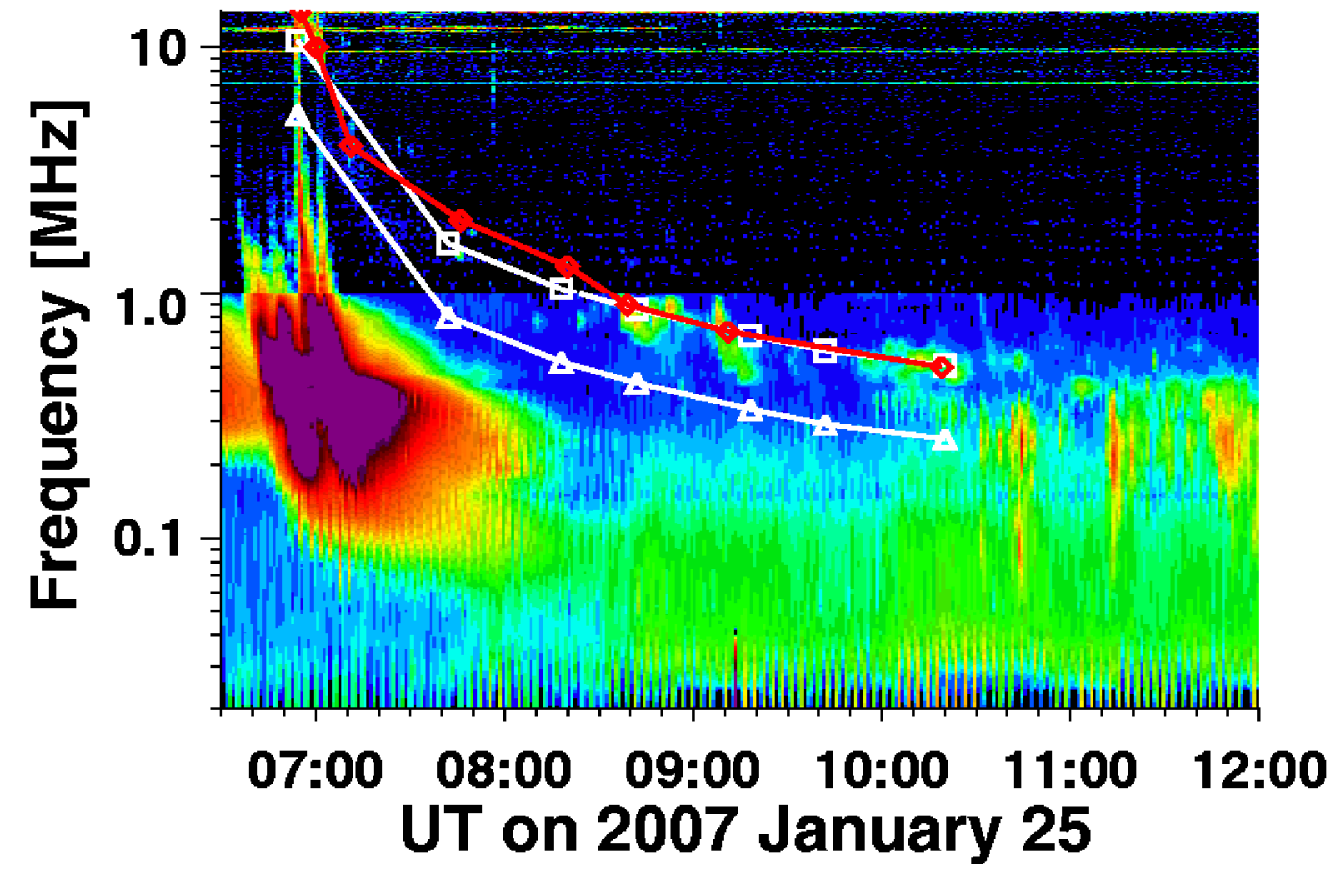

19 Figure 13. Wind/WAVES dynamic spectrum showing the type II burst with the

20 fundamental (triangles) and harmonic (squares) frequencies derived from the

21 Leblanc et al. (1998) density model superposed. The diamond symbols mark the 
1 frequency - time data points from the dynamic spectrum that were used in

2 obtaining the type II frequency corresponding to the leading edge of the CME.

4 The plasma density at a heliocentric distance of $27 \mathrm{Rs}$ can be estimated from the

5 frequency of type II burst because the radio emission takes place at the

6 fundamental or harmonic of the local plasma frequency. For this we need to

7 decide whether the observed burst is at fundamental or harmonic. Unfortunately,

8 the burst does not have both the fundamental and harmonic components, so we

9 need to resort to some models to determine the emission component. Here we use

10 the Leblanc et al. (1998) model for the variation of electron density as a function

11 of heliocentric distance. First we obtained the frequency of emission at several

12 discrete times when the radio emission is clear. Then we matched the CME

13 heights to the chosen times from the CME height-time plot (see Fig. 12). For

14 these CME heights, we can obtain the electron density (and hence the emission

15 frequency) in the IP medium using Leblanc et al. (1998) model. For each height

16 we get two frequencies, one assuming fundamental emission, and the other

17 harmonic. We thus obtain frequency - time plots, which are overlaid on the

18 dynamic spectrum in Fig. 13. The model had to be multiplied by a factor of 1.7 to

19 get the best fit to the observed variation of emission frequency with time. The

20 multiplication factor is normally obtained by looking at the plasma density at 1

$21 \mathrm{AU}$, but the present event was an east limb event, so one has to wait for a week to

22 obtain the solar wind density in the solar wind originating from the corona above

23 the eruption region. Therefore, we have chosen a multiplication factor in an ad

24 hoc manner. The frequency - time lines in Fig. 13 show that the harmonic

25 emission curve fits the observation quite well, so we conclude that the radio

26 emission takes place at the harmonic of the plasma frequency. Thus at a

27 heliocentric distance of $\sim 27 \mathrm{Rs}$, the plasma frequency is $\sim 250 \mathrm{kHz}$ (corresponding

28 to half the emission frequency), which gives a plasma density of $\sim 772 \mathrm{~cm}^{-3}$. We

29 shall make use of the radial variation of the emission frequency and the radial

30 evolution of the CME speed to understand the drift rate spectrum in section 4.

\section{$32 \quad 3.5$ Other Events}

33 Among the six remaining events, the data coverage for the 2007 February 19

34 CME was very poor: there was a LASCO data gap and COR1 observed the CME 
1 only in two frames. Moreover, the COR1 frames were obtained after the metric

2 type II burst ended. The speed computed from just two frames is not very

3 accurate. Furthermore, the eruption was near the disk center (S12E16) for both SA

4 and SB, so the measured speed is subject to large projection effects. All the three

5 events in May 2007 (19, 22, and 23) occurred in the same AR (NOAA 0956). The

6 May 19 and 22 events had COR1 images during the type II bursts. However, the

7 eruption was on the disk (N07W06 and N02W42), so the measurements were

8 subject to projection effects. The spacecraft separation was also not large enough

9 to eliminate the projection effects. The May 23 event was closer to the limb, but

10 unfortunately, the COR1 observations were not made until after the end of the

11 type II burst. The 2007 August 6 CME was associated with an impulsive flare

12 similar to the 2007 June 3 event discussed in section 3.4. Unfortunately, there was

13 no COR1 frame when the type II burst was in progress. Finally, the 2008 April 26

14 event had COR1 observations when the type II was in progress. However, the

15 eruption was on the disk for all the three views, so the height-time measurements

16 are subject to projection effects.
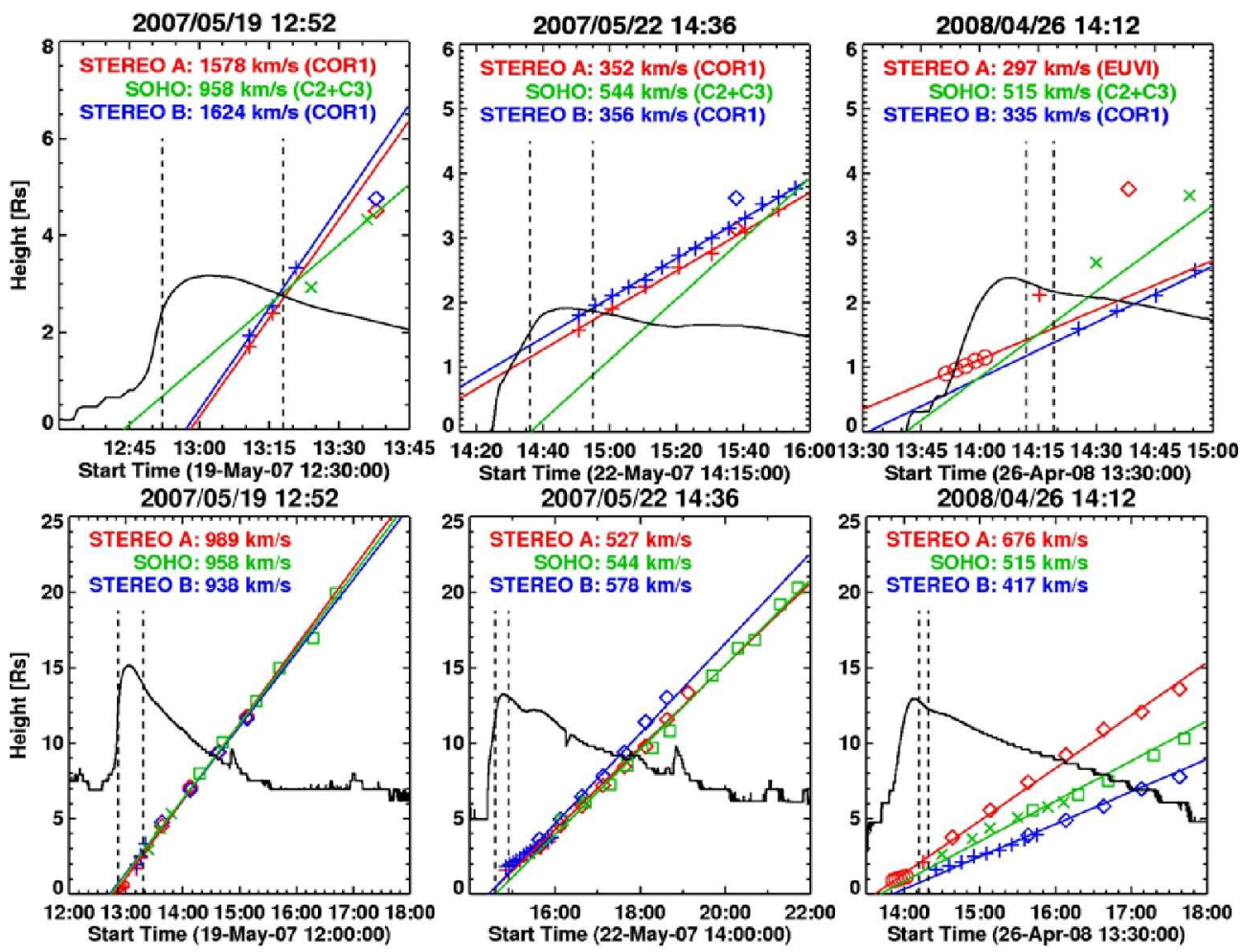

Figure 14. CME height-time plots for the events on 2007 May 19, 2007 May 22 and 2008 April 26 within the CORI FOV (top row) and the entire FOV (bottom row). For the 2008 April 26 event, there is only one CORI A data point, so we have given the speed obtained from EUVI data in the top panel. The GOES soft 
$1 X$-ray light curve in the $1-8 A$ channel and the interval of metric type II radio

2 burst (between the two vertical dashed lines) are shown for reference.

4 The height-time plots of the three events with CME data in COR1 during the

5 metric type II burst are shown in Fig. 14. The plots show that the SA, SB, and

6 SOHO plots are close to each other for the May 2007 events because the

7 spacecraft separation was not large. On the other hand, the three plots are very

8 different for the 2008 April 26 event because of the large spacecraft separation

$9\left(\sim 50^{\circ}\right)$ : SA data yielded the highest speed $(676 \mathrm{~km} / \mathrm{s})$ because the source location

10 from SA was N08E33 and the projection effects are expected to be modest. For

$11 \mathrm{SB}$, the speed is the smallest $(417 \mathrm{~km} / \mathrm{s})$ because the source location from SB

12 view is N08W15. The SOHO view had the source location at N08E09, so the

13 speed should be similar to the SB view. However, the speed is slightly higher (515

$14 \mathrm{~km} / \mathrm{s}$ ) because SOHO data points correspond to a larger height, thus missing the

15 acceleration phase of the CME. The three views to the 2008 April $26 \mathrm{CME}$ can

16 be used to get the deprojected (space) speed of the CME, but it is beyond the

17 scope of this paper.

19 4. The Universal Drift Rate Spectrum

20 We now discuss how the observational results presented above can be used to

21 understand the universal drift rate spectrum of the type II bursts over the entire

22 range of frequencies over which type II radio bursts occur. It is known for a long

23 time that the starting frequency and drift rate of metric type II bursts are well

24 correlated (see, e.g., Mann et al., 1996; Vrsnak et al., 2002). When extended to

25 the longer wavelength domain, the correlation remained high (correlation

26 coefficient $>0.8$ ). In fact over the entire frequency range of type II radio bursts in

27 the inner heliosphere, it was found that measured drift rate (df/dt) is related to the

28 emission frequency (f) by a power law (Vrsnak et al., 2001; Aguilar-Rodriguez et

29 al., 2005):

$30 \quad|\mathrm{df} / \mathrm{dt}| \sim \mathrm{f}^{\mathrm{e}}$,

31 where $\mathrm{C} \sim 2$ is the observed power-law index. The power law in (1) is valid over

32 six orders of magnitude in emission frequency, and has been shown to be true

33 even when observations from various instruments, spectral domains, and epochs 
1 are combined (Aguilar-Rodriguez et al., 2005). This is the reason we call the

2 relationship (1) as the universal drift rate spectrum.

4 Assuming that the type II radio emission occurs at the local plasma frequency

5 (fp), one can relate the speed of the disturbance away from the Sun to the drift rate

6 of the type II burst if we know how the density (n) falls off with distance (r) from

7 the Sun, where the radio emission occurs. The plasma frequency is related to the

8 plasma electron density as,

$9 \mathrm{fp}(\mathrm{r})=9 \times 10^{-3} \ln (\mathrm{r})$.

10 If we take the electron density variation with $\mathrm{r}$ as a power law,

$11 \mathrm{n}(\mathrm{r}) \sim \mathrm{r}^{-\alpha}$, we see that $\mathrm{fp}(\mathrm{r}) \sim \mathrm{r}^{-\alpha / 2}$.

12 For fundamental plasma emission, the emission frequency $f=f p$, so the radio

13 emission occurs progressively at lower frequencies at later times. Thus one can

14 write the frequency drift rate as

$15 \mathrm{df} / \mathrm{dt}=(\mathrm{df} / \mathrm{dr})(\mathrm{dr} / \mathrm{dt})$.

16 Note that $\mathrm{dr} / \mathrm{dt}=\mathrm{V}$ is the speed of the shock moving away from the Sun down the

17 density gradient. In principle the density gradient (or plasma frequency gradient)

18 may be in different direction than the direction of the disturbance. Here we

19 assume that they are in the same direction. Using equation (2) and the relation $\mathrm{f}=$

$20 \mathrm{fp}$, we can write,

$21 \mathrm{df} / \mathrm{dr}=(\mathrm{f} / 2 \mathrm{n})(\mathrm{dn} / \mathrm{dr})=(\mathrm{f} / 2 \mathrm{Ln})$,

22 where $\operatorname{Ln}=[(1 / \mathrm{n})(\mathrm{dn} / \mathrm{dr})]^{-1}$ is the density scale height in the medium through

23 which the shock propagates. Combining equations (4) and (5), we can get the

24 shock speed as

$25 \mathrm{~V}=2 \operatorname{Ln}(1 / \mathrm{f})(\mathrm{df} / \mathrm{dt}) .(6)$

26 Measuring the drift rate from the dynamic spectrum and obtaining the density

27 scale height from a density model, one can get the shock speed. The speed of the

28 shock can also be obtained from the $2 \mathrm{fp}$ (second harmonic emission) in a similar

29 fashion.

30 In order to understand the observed variation of $\mathrm{df} / \mathrm{dt}$ with $\mathrm{f}$, we need to consider

31 equation (4) together with equation (3). We can rewrite equation (4) as,

$32 \mathrm{df} / \mathrm{dt}=(\mathrm{Vf} / 2 \mathrm{n})(\mathrm{dn} / \mathrm{dr})$,

33 which upon substituting for $\mathrm{f}$ and $\mathrm{n}$ from (3) can be written as,

$34|\mathrm{df} / \mathrm{dt}| \sim \mathrm{Vr}^{-1-\alpha / 2}$. 
1 Here we have used the absolute value of $\mathrm{df} / \mathrm{dt}$ because it is negative for type II

2 bursts (frequency of emission decreases with time). Writing $r$ in terms of $f$ from

3 equation (3), we get

$4 \quad|\mathrm{df} / \mathrm{dt}| \sim \mathrm{Vf}^{(\alpha+2) / \alpha}$. (9)

5 Assuming $\alpha=2$, Vrsnak et al. (2001) obtained $d f / d t \sim V f^{2}$. Furthermore, if the

6 shock speed does not change significantly ( $V=$ constant), then

$7 \mathrm{df} / \mathrm{dt} \sim \mathrm{f}^{2}$,

8 which is close to the universal power law (see equation 1). The close relationship

9 between $\mathrm{df} / \mathrm{dt}$ and $\mathrm{f}$ can be interpreted as the property of the shock propagation in

10 the corona and IP medium. In spite of this overall agreement, there is

11 considerable deviation from $\epsilon \sim 2$ when individual frequency domains are

12 considered. Aguilar-Rodriguez et al., (2005) noted that $\epsilon$ can be as high as 2.3 in

13 the kilometric domain and as low as 1.4 in the metric domain. In the following,

14 we shall demonstrate that this variation can be explained in terms of the different

15 variations of the shock speed and the plasma density in different spatial domains

16 between the Sun and Earth.

18 Gopalswamy (2006b) pointed out that the assumption of constant $V$ may be

19 reasonable in individual wavelength domains (metric, DH or kilometric), but it

20 may not be valid over the entire wavelength range. For example, CMEs

21 associated with DH type II bursts are known to undergo rapid deceleration within

22 the SOHO/LASCO field of view (Gopalswamy et al., 2001b). We also know that

23 CMEs and shocks undergo significant change in speed between the Sun and $1 \mathrm{AU}$

24 (Gopalswamy et al., 2000) because of the drag force of the ambient medium

25 acting on the CMEs (Gopalswamy et al., 2001b; Vrsnak, 2001b). Vrsnak et al.

26 (2001) had considered the decline of the shock speed in the near-Earth IP medium

27 (kilometric domain). Here we generalize the speed variation in all the spatial

28 domains by introducing a radial dependence for $\mathrm{V}$ of the form,

$29 \mathrm{~V} \sim \mathrm{r}^{\beta}$,

30 into the relation (9). This means $\beta$ needs to be $>0$ for the metric domain and $<0$

31 for the kilometric domain in order to explain the deviations from $C \sim 2$.

32 Substituting (11) for $\mathrm{V}$ in (9) and making use of the relation (3) we get,

$33 \mathrm{df} / \mathrm{dt} \sim \mathrm{f}^{(\alpha+2 \beta+2) / \alpha}$.

34 Comparing (12) and (1) we see that, 
2 In the following we investigate the relation (8) using the observational results

3 presented in this paper.
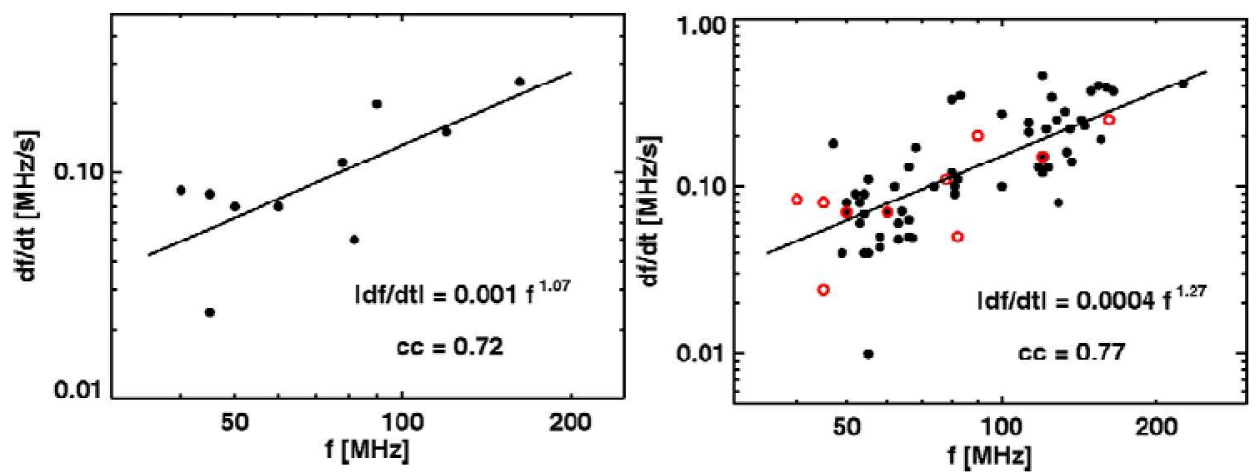

5 Figure 15. Scatter plot between the starting frequency $f$ and the drift rate $d f / d t$

6 (absolute value) for the 10 metric type II bursts (left) and a similar plot from

7 Potsdam data reported in Mann et al. (1996) with the data points from the left

8 superposed. The scatter plots represent a power law of the form $|d f / d t| \sim f^{\epsilon}$ with $C$

$9=1.07$ for the ten events and 1.27 for the 10 events combined with the 58 events

10 from Mann et al. (1996).

12 4.1 Metric Domain

13 Making use of the dynamic spectra of the 10 metric type II bursts available on line

14 (NGDC), we measured the drift rate of the fundamental components, where

15 available. Figure 15 shows a scatter plot between $\mathrm{df} / \mathrm{dt}$ and $\mathrm{f}$ for the 10 bursts.

16 The scatter plot represents a power law of the form $|\mathrm{df} / \mathrm{dt}| \sim \mathrm{f}^{1.07}$ for the ten events

17 and $|\mathrm{df} / \mathrm{dt}| \sim \mathrm{f}^{1.27}$ when the 10 events were combined with the 58 metric type II

18 bursts published by from Mann et al. (1996). Out of the 65 events published by

19 Mann et al., (1996), we chose only those events for which the drift rate of the

20 fundamental components were measured. This value of $\mathrm{C}$ is slightly smaller than

21 the value 1.44 obtained by Aguilar-Rodriguez et al. (2005). In another study of 18

22 bursts that had clear band-splitting, Vrsnak et al. (2002) obtained $\mathrm{C} \sim 1.89$, similar

23 to the universal value, but they had data to frequencies as low as $18 \mathrm{MHz}$.

25 As the observations presented in the preceding sections show, the CME speed 26 (and the shock speed) increases during the metric type II burst. The assumption 27 we make here is that the radial dependence of the shock mimics that of the CMEs. 28 This is not a bad assumption so long as the shock is driven by the CME (see, e.g., 
1 Gopalswamy et al., 2005). Therefore, we expect $\beta>0$ in (11). If we assume that

2 the heliocentric distance of the shock is not too different from that of the CME (as

3 supported by the height - time plots), we can take the CME speed to be roughly

4 the same as the shock speed for the duration of the type II bursts. We also need

5 the space speed of the CME to get the spatial dependence of $V$ (i.e., to obtain $\beta$ in

6 (11)). For this purpose, we choose only those events that are limb events from the

7 view of at least one of the spacecraft (SA, SB, or SOHO). From Table 2, we see

8 that the 2007 February and May events are all disk events for both the STEREO

9 spacecraft (the separation is not large enough). Similarly, the 2007 August event

10 and the 2008 April event are disk events for all the spacecraft (including SOHO).

11 This leaves only four limb events: January 25, June 3, and December 25 events in

122007 and the March 25 event in 2008. For the 2007 January 25 event, the CME

13 first appeared in the COR1 FOV at 06:53:25 UT at $r=2.85$ Rs. The metric type II

14 had already ended by this time, so it was not possible to detect the initial

15 acceleration of the CME. Only for the 2007 December 31 and 2008 March 25

16 CMEs we had adequate COR1 height - time measurements to derive the variation

17 of $\mathrm{V}$ as a function of $\mathrm{r}$. Of these, the 2007 December 31 had the largest number of

18 data points, so we can characterize the speed variation with height accurately.
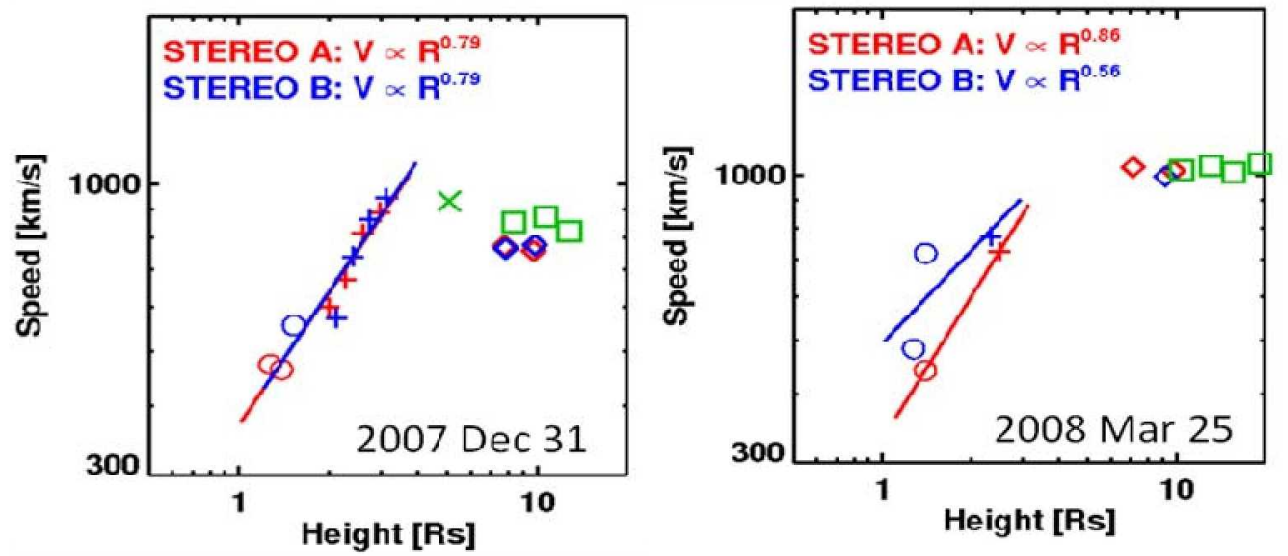

Figure 16. The variation of CME speed with height for the 2007 December 31

21 (left) and 2008 March 25 (right) events. Measurements from different instruments

22 are distinguished: circles - EUVI; plus symbols - CORI; cross symbol-

23 LASCO/C2 ; diamonds - COR2; squares: LASCO/C3. The speeds were obtained

24 using 4 successive height - time data points at each height.

25 Fig. 16 shows how V varies with $\mathrm{r}$ for the 2007 December 31 and 2008 March 25

26 events. For the December event, COR1 captured the CME in four frames (both

27 SA and SB). The CME manifestation was also observed in EUVI (two frames in 
1 SA and one frame in SB). The combined data shows that the CME starts with a

2 low speed of $\sim 450 \mathrm{~km} / \mathrm{s}$, reaches a peak speed of $\sim 1000 \mathrm{~km} / \mathrm{s}$ and then declines to

$3 \sim 700 \mathrm{~km} / \mathrm{s}$ in the outer corona. By plotting $\mathrm{V}$ as a function of $\mathrm{r}$, we find that $\mathrm{V}$

$4 \sim r^{0.79}$ for both $\mathrm{SA}$ and $\mathrm{SB}$, so $\beta=-0.79$. We now need $\alpha$ in the metric wavelength

5 domain to obtain $€$. While $\alpha \sim 2$ is applicable to the IP medium, it is well known

6 that the coronal density (where metric type II bursts occur) drops off steeply with

7 distance giving $\alpha \sim 6$ (see Saito, 1970; Leblanc et al., 1998 or data in Newkirk,

8 1967). Substituting $\alpha=6$ and $\beta=-0.79$ in eq. (13) we get $C=1.04$. This is in

9 close agreement with $\epsilon=1.07$ obtained in Fig. 15 and not too different from 1.27

10 obtained for all metric type II bursts.

12 The number of data points in the V vs. $r$ plot is small for the 2008 March 25 event.

13 Nevertheless, we were able to obtain the power law indices from SA and SB data:

$14 \beta=-0.86$ for SA and $\beta=-0.56$ for SB. Recalling the discussion in section 3.2

15 that the 2008 March 25 eruption is a good limb event only in SA view, we can

16 take the SA data to be free of projection effects. Therefore, $\beta=-0.86$ and $\alpha=6$ in

17 eq. (13) give $C=1.05$, which is similar to the value obtained from the 2007

18 December 31 event. Thus we conclude that the CME speed variation captured by

19 COR1 correctly explains the behavior of the drift rate spectrum in the metric

20 domain. A further implication of this result is the close physical connection

21 between type II radio bursts and CMEs.
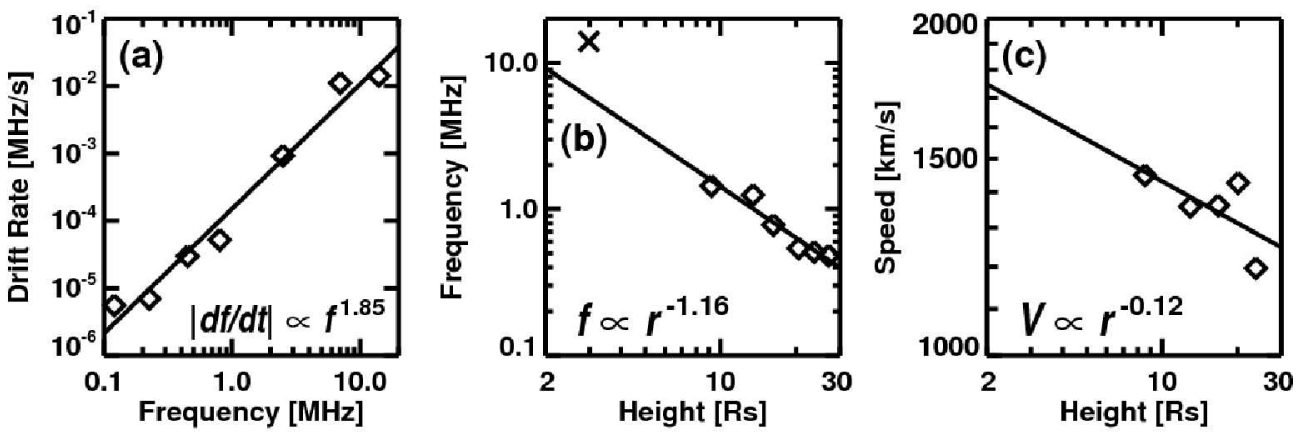

Figure 17. The drift-rate spectrum (a), the frequency variation with heliocentric

24 distance (b), and the radial variation of CME speed (c) for the 2007 January 25

25 type II burst, the only event with emission components in the kilometric domain.

26 In (b), the cross represents the $14 \mathrm{MHz}$ data point not included in the fit because it is at the transition between $\mathrm{m}$ and $\mathrm{km}$ domains. 
1 Out of the 6 type II bursts observed in the DH domain, only one extended to $\mathrm{km}$

2 wavelengths (down to $90 \mathrm{kHz}$ ). The type II burst was observed both by WAVES

3 and SWAVES. For a single event like this, we can obtain the drift rate spectrum

4 by measuring the drift rate at many segments of the dynamic spectrum as we did

5 in section 3.4. Figure 17a shows the drift rate spectrum, which has a power-law

6 index of 1.85 . This is significantly larger than the index (1.27) for the metric type

$7 \quad$ II bursts and only slightly larger than the index (1.80) for all the wavelength

8 domains. Let us see how we can obtain $E=1.85$ from eq. 13. Since we have

9 CME observations overlapping with the spatial domain of the $\mathrm{m}-\mathrm{km}$ type II burst,

10 we can directly obtain the radial dependence of the emission frequency and

11 compare it with the relation $f(r) \sim r^{-\alpha / 2}$ (relation 3 ) to get $\alpha$. For the set of times

12 at which the CME heights were measured, we obtained the corresponding

13 emission frequencies from the WAVES dynamic spectrum giving a set of

14 frequency - height data points as plotted in Fig. 17b. The scatter plot can be fit to

15 a straight line, which gives the power law index as 1.16 , which is $\alpha / 2$ according to

16 relation (3). The resulting $\alpha=2.32$ is slightly higher than the canonical value of 2

17 (see e.g., Leblanc et al, 1998; Reiner et al., 1998; Pohjolainen et al., 2007). The

18 CME observations can also be used to obtain $\beta$ by estimating the radial

19 dependence of CME speed as we did for the metric emission range. Recall from

20 section 3.4 that the frequency of emission was $\sim 500 \mathrm{kHz}$ when the $\mathrm{CME}$ reached

21 the edge of the LASCO/C3 FOV, overlapping with the spatial domain

22 corresponding to the $\mathrm{km}$ wavelengths. We computed the radial dependence of $\mathrm{V}$

23 using three height - time data points at a time. Figure $17 \mathrm{c}$ shows that the $\mathrm{V}$

24 declines with $r$ characterized by a power law index $\beta=0.12$. Note that $\beta$ is now

25 positive, opposite to what we found in the inner corona (see fig. 16). Using $\alpha=$

262.32 and $\beta=0.12$ in equation (13), we get $\Theta=1.97$, in good agreement with the

27 value (1.85) obtained from the radio dynamic spectrum. If we use the canonical

28 value of $\alpha=2$, we get $C=2.12$. Vrsnak et al. (2001) used shock speed as a

29 function of heliocentric distance inferred from density models and obtained $\beta=$

$30 \quad 0.13$, which is consistent with the value (0.12) obtained here using CME

31 observations. As noted before, we have tacitly assumed that the shock speed is the

32 same as the CME speed. We thus conclude that the drift rate spectrum at

33 kilometric wavelengths is steeper than that in the metric wavelengths because of

34 the opposite behavior of speed as a function of heliocentric distance. 


\section{5. Discussion and Summary}

2 The inner coronagraph COR1 on board STEREO has a field of view covering 1.4

3 to 4 Rs, which enables observing CMEs close to the onset of type II bursts. EUV

4 waves were found in most of the cases well before the start of the type II bursts,

5 indicating that the fast-mode wave around the CME becomes a shock when the

6 CME becomes super-Alfvenic. In all the EUVI snapshots obtained during the

7 metric type II bursts the EUV disturbance was found to match with the outermost

8 edge of the white-light disturbance, suggesting that the CME is the driver of the

9 EUV wave, which becomes a shock to produce the type II burst.

11 We were able to determine the speed evolution of CMEs in the inner corona and

12 the speed profile has some similarity to the Alfven speed profile at least for the

13 two well observed events (2007 December 31 and 2008 March 25). It is fairly

14 clear that the type II burst starts at a height where the Alfven speed is minimum in

15 the corona (see Fig. 5). But the height at which the type II burst ends depends on

16 the relative variation of the CME speed and the coronal Alfven speed. In the three

17 cases discussed extensively, the type II ends when the CME reaches a heliocentric

18 distance where the Alfven speed peaks. The type II burst seems to end when the

19 shock becomes subcritical or dissipates as an MHD wave in the high-Alfven

20 speed region. In two events, the speed had an impulsive behavior, very similar to

21 that of the soft X-ray profiles. The radio emission ended even before the height at

22 which the Alfven speed reached its peak value. Zhang et al. (2001) reported a

23 CME associated with the impulsive flare of 1997 May 16 at 12:54 UT (duration

$24 \sim 15 \mathrm{~min}$ ). However, Zhang et al. (2001) did not find the CME speed behavior to

25 follow the X-ray spike. A detailed examination of soft X-ray images from Yohkoh

26 revealed a post-eruption arcade and the flare actually was of long duration with

27 the impulsive spike superposed on it. The long duration flare started around 12:10

28 UT on 1997 May 16 and continued beyond 16 UT. Zhang et al.'s CME speed

29 profile followed this long duration flare rather than the impulsive spike. In

30 contrast, the CME speed profile in our case followed the impulsive spike.

32 For the three events in which we were able to measure the CME height at the time

33 of the metric type II burst onset, we found that the CME leading edge was at

34 heights of $\sim 1.39,1.5$, and $1.66 \mathrm{Rs}$ (average $1.55 \mathrm{Rs}$ ). This average value is 
1 remarkably similar to the height at which the coronal Alfven speed attains a

2 minimum value ( 1.4 Rs, see Fig. 5) between the active region and the quiet

3 coronal values (Gopalswamy et al., 2001a; Mann et al., 2003). This is

4 significantly lower than the corresponding CME height (2.2 Rs) obtained from

5 LASCO data alone. One can think of two reasons for this difference: (i) the

6 LASCO observations do not capture the initial speed variation of the CME and

7 thus correspond to higher average speeds; (ii) the plasma level in the corona for a

8 given frequency may shift to larger or smaller heliocentric distances, depending

9 on the phase of the solar cycle. It has been reported that the coronal density

10 during the solar maximum is higher by a factor of 4 than that in the declining

11 phase (MacQueen et al., 2001). Since the type II bursts in this paper correspond to

12 the declining phase of solar cycle 23 , we expect that a given plasma level would

13 move closer to the Sun compared to the solar maximum phase. Cliver et al.,

14 (2004) reported a metric type II burst on 1997 November 6 with CME observation

15 available (from LASCO/C1) close to the onset of the burst; they estimated the

16 CME height to be $\sim 1.33$ Rs at metric type II onset. Since this event occurred

17 during the minimum phase it is consistent with our conclusion that the low CME

18 height at type II start may be due to the solar cycle effect. The density variation

19 over the solar cycle may also have an influence on the radial profile of the Alfven

20 speed, but this needs a careful consideration including the magnetic field variation

21 in the corona.

22 We also set out to understand the deviations from the power-law nature of the

23 drift rate spectrum of type II radio bursts. The deviations are the decrease in the

24 power law index at metric wavelengths and the increase in the km wavelength

25 domain with respect to the overall value of 2 corresponding to the entire

26 wavelength domain (metric to kilometric wavelengths). We provided

27 observational evidence to show that these deviations are a direct consequence of

28 the CME speed increase in the inner corona and decrease in the IP medium. While

29 the speed decrease in the IP medium was quantified from shock speeds inferred

30 from density models (Vrsnak et al., 2001), we have obtained the speed variation

31 from CME data. In addition, we found that the speed variation in the inner corona

32 is opposite to that from the IP medium and can be directly linked to the positive

33 acceleration of CMEs. 
1 In summary, we were able to show that MHD fast mode shocks form very low in

2 the corona, when the CME leading edge is only 1.5 Rs from the Sun center using

3 data from the inner coronagraph (COR1) on board the STEREO mission. This

4 distance also coincides with the spatial domain where the coronal Alfven speed

5 attains a minimum value. How the shock survives beyond this point depends on

6 the relative increase in CME speed and the Alfven speed. The CME speed and the

7 Alfven speed seem to reach their peak values around the 3-4 Rs in the corona,

8 and many type II bursts end around this time because the associated shocks

9 become subcritical or dissipate. When the CME speed is much higher than the

10 peak Alfven speed, then it can drive the shock far into the IP medium resulting in

11 the interplanetary type II burst, as was the case for the 2007 January 25 event. It is

12 possible that the CME height at the time of the type II burst onset depends on the

13 phase of the solar cycle, but this needs further investigation. While the speed in

14 the inner corona increases due to the propelling force, the CME speed decreases in

15 the IP medium due to the drag force. This opposite tendency in CME speed

16 variation can be quantified as a power law with positive (inner corona) and

17 negative (IP medium) indices that naturally explain the deviations of the universal

18 drift-rate spectrum of type II bursts. Such an explanation is consistent with the

19 CME driving the shock irrespective of the spectral domain in which type II bursts

20 are observed.

21 Acknowledgements

22 The SECCHI instrument was constructed by a consortium of international

23 institutions: the Naval Research Laboratory (USA), the Lockheed Martin

24 Solar and Astrophysical Laboratory (USA), the NASA Goddard Space

25 Flight Center (USA), the Max Planck Institut fur Sonnensystemforschung

26 (Germany), the Centre Spatial de Liege (Belgium), the University of Birmingham

27 (UK), the Rutherford Appleton Laboratory (UK), the Institut d'Optique (France),

28 and the Institute d'Astrophysique Spatiale (France). We thank the anonymous

29 referee for helpful comments.

\section{$31 \quad$ References}

32 Aguilar-Rodriguez, E., Gopalswamy, N., MacDowall, R. J., Yashiro, S., and

33 Kaiser, M. L.: 2005, in Proceeding of Solar Wind 11/SOHO 16, p. 393.

34 Bougeret, J.-L., et al.: 1995, Space Sci. Rev., 71, 231.

35 Bougeret, J. L., et al.: 2008, Space Sci. Rev., 136, 487. 
1 Brueckner, G. E., Howard, R. A., Koomen, M., et al.: 1995, Solar Phys., 162, 357.

2 Cliver, E.W., Nitta, N. V., Thompson, B. J., and Zhang, J.: 2004, Solar Phys., 225 , 3105.

4 Gopalswamy, N.: 2006a, Journal of Astrophysics and Astronomy, 27, 243.

5 Gopalswamy, N.: 2006b, AGU Geophysical Monograph, Vol. 165, American

6 Geophysical Union, Washington, DC, p. 207.

7 Gopalswamy, N. and Thompson, B. J.: 2000, J. Atmos. Sol. Terr. Phys., 62, 1457.

8 Gopalswamy, N. and Kaiser, M. L.: 2002, Adv. Space Res., 29(3), 307.

9 Gopalswamy, N., Lara, A., Lepping, R. P., Kaiser, M. L., Berdichevsky, D. and

10 St. Cyr, O. C.: 2000, Geophys. Res. Lett., 27, 145.

11 Gopalswamy, N., Lara, A., Kaiser, M. L., Bougeret, J.-L.: 2001a, J. Geophys.

12 Res., 106, 25261.

13 Gopalswamy, N., Yashiro, S., Kaiser, M. L., Howard, R. A., Bougeret, J.-L.:

142001 b, J. Geophys. Res., 106, 29219.

15 Gopalswamy, N., Aguilar-Rodriguez, E., Yashiro, S., Nunes, S., Kaiser, M. L., 16 and Howard, R. A.: 2005, J. Geophys. Res., 110, A12S07.

17 Gopalswamy, N., Yashiro, S., Xie, H., Akiyama, S., Aguilar-Rodriguez, E., 18 Kaiser, M.L., Howard, R.A., and Bougeret, J.-L.: 2008a, Astrophys. J., 674, 560.

19 Gopalswamy, N., Yashiro, S., Akiyama, S., Mäkelä, P., Xie, H., Kaiser, M. L., 20 Howard, R. A., and Bougeret, J.-L.: 2008b, AnnGeo, 26, 3033.

21 Holman, G.D. and Pesses, M. E.: 1983, Astrophys. J., 267, 837.

22 Howard, R. A., et al.: 2008, Space Sci. Rev., 136, 67.

23 Leblanc, Y., Dulk, G. A., and Bougeret, J.-L.: 1998, Sol. Phys., 183, 165.

24 Mann, G., Klassen, A., Classen, H. T., Aurass, H., Scholz, D., MacDowall, R. J., 25 Stone, R. G.: 1996, Astron. Astrophys., 119, 489.

26 Mann, G., Klassen, A., Estel, C., and Thompson, B. J.: 1999: in Proc. of 8th

27 SOHO Workshop, Edited by J.-C. Vial and B. Kaldeich-Schmann, p. 477.

28 Mann, G., Klassen, A., Aurass, H. Classen, H.-T.: 2003, A\&A, 400, 329.

29 MacQueen, R. M., Burkepile, J. T., Holzer, T. E., Stanger, A. L., and Spence, K.

30 E.: 2001, Astrophys. J., 549, 1175.

31 Michalek, G., Gopalswamy, N., and Xie, H.: 2007, Solar Phys., 246, 409.

32 Newkirk, G. A.: 1967, Ann. Rev. Astron. Astrophys., 5, 213.

33 Pohjolainen, S., van Driel-Gesztelyi, L., Culhane, J. L., Manoharan, P. K., 34 Elliott, H. A.:2007, Solar Phys., 244, 167.

35 Reiner, M. J., Kaiser, M. L., Fainberg, J., and Stone, R. G.: 1998, J. Geophys.

36 Res., 103, 29651.

37 Saito, K.: 1970, Ann. Tokyo Astron. Obs. Ser 2, 12, 53.

38 Saito, K., Poland, A. I., and Munro, R. H.: 1977, Solar Phys., 55, 121.

39 Sheeley et al.: 1997, Astrophys. J., 484, 472. 
1 Thompson, B. J., Cliver, E. W., Nitta, N., Delannée, C., and Delaboudinière, J.-P.:

2 2000, Geophys. Res. Lett., 27, 1431.

3 Vrsnak, B.: 2001, Solar Phys., 202, 173.

4 Vrsnak, B., Aurass, H., Magdalenic, J., and Gopalswamy, N.: 2001, Astron.

5 Astrophys., 377, 321.

6 Vršnak, B., Magdalenić, J., Aurass, H., Mann, G.: 2002, Astron. Astrophys., 396, 7673.

8 Vršnak, B. and Cliver, E.: 2008, Solar Phys., 253, 215.Wood, B. E. et al.: 1999, 9 Astrophys. J., 512, 484.

10 Zhang, J., Kundu, M. R., White, S. M., Dere, K. P., and Newmark, J. S.: 2001, 11 Astrophys. J., 561, 396. 